九州大学学術情報リポジトリ

Kyushu University Institutional Repository

\title{
$\mathrm{N}$-soliton solutions to the DKP equation and Weyl group actions
}

Kodama, Yuj i

Department of Mathematics, Ohio State University

Maruno, Ken-Ichi

Faculty of Mathematics, Kyushu University

http://hdl. handle. net/2324/11860

出版情報: Journal of Physics A : Mathematical and General. 39 (15), pp.4063-4086, 2006-03-29. Institute of Physics

バージョン：

権利関係 : 


\title{
MHF Preprint Series
}

Kyushu University

21st Century COE Program

Development of Dynamic Mathematics with

High Functionality

\section{N-Soliton solutions to the DKP equation and Weyl group actions}

\author{
Y. Kodama \& K. Maruno
}

MHF 2006-7

( Received March 1, 2006 )

Faculty of Mathematics

Kyushu University

Fukuoka, JAPAN 


\title{
$N$-SOLITON SOLUTIONS TO THE DKP EQUATION AND WEYL GROUP ACTIONS
}

\author{
YUJI KODAMA* AND KEN-ICHI MARUNO ${ }^{\dagger}$
}

Abstract. We study soliton solutions to the DKP equation which is defined by the Hirota bilinear form,

$$
\left\{\begin{array}{l}
\left(-4 D_{x} D_{t}+D_{x}^{4}+3 D_{y}^{2}\right) \tau_{n} \cdot \tau_{n}=24 \tau_{n-1} \tau_{n+1}, \quad n=1,2, \ldots \\
\left(2 D_{t}+D_{x}^{3} \mp 3 D_{x} D_{y}\right) \tau_{n \pm 1} \cdot \tau_{n}=0
\end{array}\right.
$$

where $\tau_{0}=1$. The $\tau$-functions $\tau_{n}$ are given by the pfaffians of certain skew-symmetric matrix. We identify one-soliton solution as an element of the Weyl group of D-type, and discuss a general structure of the interaction patterns among the solitons. Soliton solutions are characterized by $4 N \times 4 N$ skew-symmetric constant matrix which we call the $B$-matrices. We then find that one can have $M$-soliton solutions with $M$ being any number from $N$ to $2 N-1$ for some of the $4 N \times 4 N$ $B$-matrices having only $2 N$ nonzero entries in the upper triangular part (the number of solitons obtained from those $B$-matrices was previously expected to be just $N$ ).

\section{INTRODUCTION}

We study soliton solutions of the DKP equation $[1,3,9]$,

$$
\left\{\begin{array}{l}
\left(-4 D_{x} D_{t}+D_{x}^{4}+3 D_{y}^{2}\right) \tau_{n} \cdot \tau_{n}=24 \tau_{n-1} \tau_{n+1}, \\
\left(2 D_{t}+D_{x}^{3} \mp 3 D_{x} D_{y}\right) \tau_{n \pm 1} \cdot \tau_{n}=0
\end{array} \quad n=1,2 \ldots,\right.
$$

where $\tau_{0}=1$, and $D_{x}, D_{y}$ and $D_{t}$ are the usual Hirota derivatives. For each $n$, the variables $u=2\left(\ln \tau_{n}\right)_{x x}$ and $v^{ \pm}=\tau_{n \pm 1} / \tau_{n}$ define the coupled KP equation (see for example [3]),

$$
\left\{\begin{array}{l}
\left(-4 u_{t}+u_{x x x}+6 u u_{x}\right)_{x}+3 u_{y y}=24\left(v^{+} v^{-}\right)_{x x}, \\
2 v_{t}^{ \pm}+v_{x x x}^{ \pm}+3 u v_{x}^{ \pm} \mp 3\left(v_{x y}^{ \pm}+v^{ \pm} \int^{x} u_{y} d x\right)=0 .
\end{array}\right.
$$

This set of equations admits a class of particular solutions, called soliton solutions similar to those of the KP equation (see for example [5]), and in this paper we describe some properties of those solutions.

Remark 1.1. The DKP equation was given as a first member of the DKP hierarchy in [6]. In [3], the coupled KP equation was introduced as an extension of the KP equation whose solutions are given by the pfaffian form. The DKP equation was rediscovered as the Pfaff lattice describing the partition function of a skew-symmetric matrix model in [1, 7], and was also found as an orbit of some infinite-dimensional Clifford group action in [9].

The $\tau_{n}$ functions are given by the pfaffians of $2 n \times 2 n$ skew-symmetric matrices $\mathcal{Q}_{n}$ whose entries are denoted by $Q_{i, j}$ for $1 \leq i<j \leq 2 n$ with $Q_{j, i}=-Q_{i, j}$,

$$
\tau_{n}=\operatorname{Pf}\left(\mathcal{Q}_{n}\right)=\sum_{\substack{1=i_{1}<\ldots<i_{n}<2 n \\ i_{k}<j_{k}, k=1, \ldots, n}} \sigma\left(i_{1}, j_{1}, \ldots, i_{n}, j_{n}\right) Q_{i_{1}, j_{1}} Q_{i_{2}, j_{2}} \cdots Q_{i_{n}, j_{n}}
$$

*Partially supported by NSF grant DMS0404931. † Supported by COE program at Faculty of Mathematics, Kyushu University. 
Each coefficient $\sigma\left(i_{1}, j_{1}, \ldots, i_{n}, j_{n}\right)$ gives a sign corresponding to the parity of the permutation,

$$
\sigma:=\operatorname{sign}\left(\begin{array}{ccccc}
1 & 2 & \cdots & 2 n-1 & 2 n \\
i_{1} & j_{1} & \cdots & i_{n} & j_{n}
\end{array}\right)
$$

Here the elements $Q_{i, j}$ satisfy

$$
\frac{\partial}{\partial t_{k}} Q_{i, j}=Q_{i+k, j}+Q_{i, j+k}, \quad k=1,2, \ldots
$$

where $t_{1}=x, t_{2}=y, t_{3}=t$ and others are the symmetry parameters. A realization of $Q_{i, j}$ is given by [3],

$$
Q_{i, j}=\left|\begin{array}{ll}
\phi^{(i-1)} & \phi^{(j-1)} \\
\psi^{(i-1)} & \psi^{(j-1)}
\end{array}\right|, \quad \text { for } \quad i<j
$$

where $\phi^{(k)}=\partial^{k} \phi / \partial x^{k}$ (the same for $\psi^{(k)}$ ) and the functions $\phi$ and $\psi$ satisfy the same equations for $x$ and $t$ evolutions,

$$
\frac{\partial \phi}{\partial y}=\frac{\partial^{2} \phi}{\partial x^{2}}, \quad \frac{\partial \phi}{\partial t}=\frac{\partial^{3} \phi}{\partial x^{3}} .
$$

For an example of finite dimensional solution, we consider

$$
\phi(x, y, t)=\sum_{m=1}^{M} a_{m} E_{m}(x, y, t), \quad \psi(x, y, t)=\sum_{m=1}^{M} b_{m} E_{m}(x, y, t),
$$

with some constants $a_{m}, b_{m}$ for $m=1, \ldots, M$. The function $E_{m}(x, y, t)$ is the exponential function,

$$
E_{m}(x, y, t):=e^{\theta_{m}} \quad \text { with } \quad \theta_{m}(x, y, t)=p_{m} x+p_{m}^{2} y+p_{m}^{3} t+\theta_{m}^{0},
$$

where $p_{m}$ and $\theta_{m}^{0}$ are arbitrary constants, and throughout this paper we assume the parameters $\mathbf{p}:=\left(p_{1}, p_{2}, \ldots, p_{M}\right)$ to be ordered as,

$$
p_{1}<p_{2}<\cdots<p_{M} .
$$

With those $\phi$ and $\psi$, the elements $Q_{i, j}, 1 \leq i<j \leq 2 n$ become

$$
\begin{aligned}
Q_{i, j} & =\sum_{1 \leq k<l \leq M} b_{k, l}\left|\begin{array}{ll}
E_{k}^{(i-1)} & E_{k}^{(j-1)} \\
E_{l}^{(i-1)} & E_{l}^{(j-1)}
\end{array}\right| \\
& =\sum_{1 \leq k<l \leq M} b_{k, l}\left(p_{k} p_{l}\right)^{i-1}\left(p_{l}^{j-i}-p_{k}^{j-i}\right) E_{k, l},
\end{aligned}
$$

where $b_{k, l}=a_{k} b_{l}-a_{l} b_{k}$, and $E_{k, l}:=E_{k} E_{l}=\exp \left(\theta_{k}+\theta_{l}\right)$.

As a generalization of this form of $Q_{i, j}$, we consider an arbitrary $M \times M$ skew-symmetric matrix $B=\left(b_{k, l}\right)_{1 \leq k, l \leq M}$. We then note that the $2 n \times 2 n$ matrix $\mathcal{Q}_{n}$ in the $\tau$-function $\tau_{n}$ can be expressed as

$$
\mathcal{Q}_{n}(x, y, t)=\mathcal{E}_{n}(x, y, t) B \mathcal{E}_{n}(x, y, t)^{T}
$$

where $\mathcal{E}_{n}$ is a $2 n \times M$ matrix with its transpose $\mathcal{E}_{n}^{T}$, and it is given by

$$
\mathcal{E}_{n}=\left(\begin{array}{cccc}
E_{1} & E_{2} & \cdots & E_{M} \\
\vdots & \vdots & \ddots & \vdots \\
E_{1}^{(2 n-1)} & E_{2}^{(2 n-1)} & \cdots & E_{M}^{(2 n-1)}
\end{array}\right) .
$$

In this paper, we discuss a classification problem of several soliton solutions given by (1.6) in terms of the $B$-matrix. Note here that $M>2$ for $n \geq 1$, since the case with $M=2$ gives a trivial solution, i.e. $\tau_{1}=Q_{1,2}=b_{1,2}\left(p_{2}-p_{1}\right) E_{1} E_{2}$ and $u=2\left(\ln \tau_{1}\right)_{x x}=0$. Also note that one needs $M>2 n$ for $u=2\left(\ln \tau_{n}\right)_{x x} \neq 0$.

One should also note that if $\tau_{n+1}=0$ (i.e. $v^{+}=0$ ), the corresponding solution satisfies the $\mathrm{KP}$ equation. This condition can be of course obtained from the structure of the $B$-matrix. For example, if we take $M=3$, then $\tau_{2}$ vanishes identically (the size of the pfaffian is $4 \times 4$, but the independent exponentials are three or less). This implies that $\tau_{1}$ with $M=3$ gives a solution of the 
$\mathrm{KP}$ equation, and it gives either one KP soliton solution or a resonant Y-shape KP soliton: With a $3 \times 3 B$-matrix, we have

$$
\tau_{1}=Q_{1,2}=b_{1,2}\left(p_{2}-p_{1}\right) E_{1} E_{2}+b_{1,3}\left(p_{3}-p_{1}\right) E_{1} E_{3}+b_{2,3}\left(p_{3}-p_{2}\right) E_{2} E_{3} .
$$

The function $u=2\left(\ln \tau_{1}\right)_{x x}$ gives one KP soliton solution if one of $b_{i, j}$ is zero (with others being positive), and Y-shape KP soliton if all $b_{i, j}$ are positive. For example, with $b_{1,2}=0$, we have $\tau_{1}=\left(b_{1,3}\left(p_{3}-p_{1}\right) E_{1}+b_{2,3}\left(p_{3}-p_{2}\right) E_{2}\right) E_{3}$ which leads to

$$
w(x, y, t):=\frac{\partial}{\partial x} \ln \tau_{1}=p_{3}+\frac{1}{2}\left(p_{1}+p_{2}\right)+\frac{1}{2}\left(p_{2}-p_{1}\right) \tanh \frac{1}{2}\left(\theta_{2}-\theta_{1}\right) .
$$

The asymptotic values of $w$ then take

$$
w(x, y, t) \rightarrow\left\{\begin{array}{lll}
p_{1}+p_{3}, & \text { for } & x \rightarrow-\infty \\
p_{2}+p_{3}, & \text { for } & x \rightarrow \infty
\end{array}\right.
$$

Note here that the one soliton exchange the asymptotic values of $(1,3):=p_{1}+p_{3}$ and $(2,3):=p_{2}+p_{3}$, that is, this one soliton permutes the numbers, $(1) \leftrightarrow(2)$. We then label one soliton of the KP equation as an element of the permutation group $W$, in this case $W=S_{3}$, the symmetry group of order 3. We denote the one soliton solution (1.7) by [1:2], and call this type of soliton A-soliton ("A" stands for type A Lie algebra which is the underlying symmetry algebra for KP equation). In general, we denote one A-soliton by $[i: j]$, if the function $w$ exchanges $p_{i} \leftrightarrow p_{j}$ with $p_{i}<p_{j}$. We sometime identify $[i: j]$ as an element of the symmetry group.

A generic solution of the DKP equation is then obtained for $M \geq 4$. In particular, we obtain one soliton solution in the case $M=4$ with the $B$-matrix having just two nonzero elements in the upper triangular part. For example, we consider the $B$-matrix having $b_{1,2}=1, b_{3,4}=1$ and all others $b_{i, j}=0$ for $i<j$. Then

$$
\tau_{1}=Q_{1,2}=\left(p_{2}-p_{1}\right) E_{1} E_{2}+\left(p_{4}-p_{3}\right) E_{3} E_{4}
$$

This gives

$$
w(x, y, t):=\frac{\partial}{\partial x} \ln \tau_{1}=\frac{1}{2} \sum_{k=1}^{4} p_{k}+\frac{1}{2}\left(p_{3}+p_{4}-p_{1}-p_{2}\right) \tanh \frac{1}{2}\left(\theta_{34}-\theta_{12}\right)
$$

with $\theta_{i j}=\theta_{i}+\theta_{j}+\ln \left|p_{i}-p_{j}\right|$. For each asymptotic of $x \rightarrow \pm \infty$, one of the exponential terms in the $\tau$-function becomes dominant. In the case of (1.8), we have

$$
w(x, y, t) \rightarrow \begin{cases}p_{1}+p_{2}, & \text { as } x \rightarrow-\infty \\ p_{3}+p_{4}, & \text { as } x \rightarrow \infty\end{cases}
$$

Thus, this one D-soliton exchanges $(1,2) \leftrightarrow(3,4)$ with the values of $w$ where $(i, j):=p_{i}+p_{j}$. This indicates a Weyl-action of $D$-type: For example, $\pi_{1,2} \in W^{D}$ may be expressed as

$$
\pi_{1,2} \cdot\left(i_{1}, i_{2}: i_{\overline{1}}, i_{\overline{2}}\right)=\left(i_{2}, i_{1}: i_{\overline{2}}, i_{\overline{1}}\right), \quad i_{1}=1, i_{2}=3, i_{\overline{1}}=2, i_{\overline{2}}=4 .
$$

We denote this D-soliton by $[1,2: 3,4]$. Then identifying $[1,2: 3,4]$ with the permutation of the pairs, $(1,2) \leftrightarrow(3,4)$, one has the relation $[1,2: 3,4]=[1: 3] \cdot[2: 4]=[1: 4] \cdot[2: 3]$ where $[i: j]$ is the permutation $(i) \leftrightarrow(j)$ of A-soliton. This relation implies a resonant bifurcation of one D-soliton into two A-solitons (see Section 4). Since one D-soliton has four $p_{i}$-parameters, we need to have $M=4 N$ parameters to describe $N$ D-soliton solution as a solution given by the $\tau$-function $\tau_{N}$. However, we show in this paper that $4 N$ parameter solution can contain up to $2 N-1$ number of D-solitons (this is quite different from the solitons of the KP equation).

In this paper, we study soliton solution of the DKP equation consisting of those A- and D-type soliton solutions. In Section 2, we give a general structure of the $\tau$-functions. Then in Section 3 , we discuss some details of one D-soliton solutions given by the $B$-matrix of size $4 \times 4$. Here we also classify the soliton solutions obtained by $4 \times 4 B$-matrices. In Section 4 , we present the soliton solutions for the case of $8 \times 8 B$-matrices, and classify the soliton solutions consisting of only D-types. It turns out that the case with $8 \times 8 B$-matrix can have either two or three D-solitons depending on the values of the parameters $\left\{p_{i}: i=1 \ldots 8\right\}$. The generic solution given by the $B$-matrix having 
all nonzero entries is then given by four A-solitons. Finally we discuss the general case of $4 N \times 4 N$ $B$-matrices, i.e. $M=4 N$, in Section 5. Then we show that the number of D-solitons for those $B$-matrices having $2 N$ nonzero entries in the upper triangular part can be any number from $N$ to $2 N-1$, contrary to the previous study (see [5]) where the number of D-soliton is expected to be just $N$.

In [8], we showed that the $N$-soliton solutions of the KP equation can be classified by the Schubert decomposition of the Grassmannian $\operatorname{Gr}(N, 2 N)$. We also expect that $N$-soliton solutions of the DKP equation can be classified by the similar decomposition of the orthogonal Grassmannain $\operatorname{OGr}(M, 2 M)$ for some $M$. However, in this paper, we just present elementary feature of the $N$-soliton solutions based on the pfaffian structure of the $\tau$ function, which is already complicated but has several interesting aspects. We plan to discuss a classification problem based on the geometric structure of the orthogonal Grassmannain in a future communication.

\section{Structure of the $\tau$-Functions}

We first note that the $\tau$-function (1.2) with the $\mathcal{Q}_{n}$ matrix (1.6) has the following expansion theorem, i.e. the pfaffian version of the Binet-Cauchy theorem (see [4] for the details):

Lemma 2.1. The $\tau$-functions of (1.2) with (1.6) can be expressed by

$$
\tau_{n}=\sum_{1 \leq i_{1}<\cdots<i_{2 n} \leq 4 N} \operatorname{Pf}\left(B\left(i_{1}, \ldots, i_{2 n}\right)\right) \operatorname{Det}\left(E\left(i_{1}, \ldots, i_{2 n}\right)\right) .
$$

where $E\left(i_{1}, \ldots, i_{2 n}\right)$ is $2 n \times 2 n$ submatrix of the $2 n \times 4 N$ matrix $\mathcal{E}_{n}$,

$$
E\left(i_{1}, \ldots, i_{2 n}\right):=\left(\begin{array}{ccc}
E_{i_{1}} & \cdots & E_{i_{2 n}} \\
\vdots & \ddots & \vdots \\
E_{i_{1}}^{(2 n-1)} & \cdots & E_{i_{2 n}}^{(2 n-1)}
\end{array}\right)
$$

and $B\left(i_{1}, \ldots, i_{2 n}\right)$ is $2 n \times 2 n$ skewsymmetric submatrix of the $4 N \times 4 N$ matrix $B$,

$$
B\left(i_{1}, \ldots, i_{2 n}\right)=\left(\begin{array}{ccccc}
0 & b_{i_{1}, i_{2}} & \cdots & \cdots & b_{i_{1}, i_{2 n}} \\
& 0 & \ddots & \cdots & b_{i_{2}, i_{2 n}} \\
& & \ddots & \ddots & \vdots \\
& & & 0 & b_{i_{2 n-1}, i_{2 n}} \\
& & & & 0
\end{array}\right)
$$

The lower triangular part is given by $b_{i_{l}, i_{k}}=-b_{i_{k}, i_{l}}$, and is left in blank. Also Det $(E)$ is given by the Wronskian determinant,

$$
\operatorname{Det}\left(E\left(i_{1}, \cdots, i_{2 n}\right)\right)=\operatorname{Wr}\left(E_{i_{1}}, \ldots, E_{i_{2 n}}\right)=\prod_{m<n}\left(p_{i_{n}}-p_{i_{m}}\right) \exp \left(\sum_{j=1}^{2 n} \theta_{i_{j}}\right)>0
$$

The sign is due to the order (1.4), i.e. $p_{1}<p_{2}<\cdots<p_{4 N}$.

From this formula of the $\tau$ function, the soliton solutions are completely determined by the nonzero coefficients of the pfaffians $\operatorname{Pf}\left(B\left(i_{1}, \ldots, i_{2 n}\right)\right)$ which are the Plücker coordinates of the orthogonal Grassmannan $\operatorname{Gr}(2 n, 4 N)$. For example, if all the coefficients are positive, then we have $(2 n, 4 N-2 n)-$ soliton solution in $u(x, y, t)$, that is, we have $2 n$ out-going line solitons in $y \rightarrow \infty$ and $4 N-2 n$ in-coming line solitons in $y \rightarrow-\infty$ (see [2]). As we can see from [8] that in particular, if $n=N$ we have an $2 N$-soliton solution similar to the KP equation, i.e. all $2 N$ solitons are A-solitons, and all the interactions are of T-types. Then the main purpose of this paper is to identify soliton solution of the DKP equation with a certain combination of nonzero coefficients.

Example 2.1. As the simplest example, the pfaffian $\operatorname{Pf}\left(B\left(i_{1}, \ldots, i_{4}\right)\right)$ is given by

$$
\operatorname{Pf}(B(1, \ldots, 4))=b_{1,2} b_{3,4}-b_{1,3} b_{2,4}+b_{1,4} b_{2,3} .
$$


Note here that if the indices in each term have partial overlap, then the product takes the minus sign, e.g. $(1,3)$ and $(2,4)$. This is a key for the classification of four A-solitons (see Section 4).

From Lemma 2.1, one should note that each term in $\tau_{n}$ in (1.2) is given by the product of $2 n$ exponentials $E_{k}$, i.e.

$$
\prod_{j=1}^{2 n} E_{i_{j}}=\prod_{k=1}^{n} E_{i_{k}, j_{k}}
$$

where $\left\{i_{j} \mid j=1, \ldots, 2 n\right\}=\left\{i_{k}, j_{k} \mid i_{k}<j_{k}, k=1, \ldots, n\right\}$, and they are the indices for the elements of the $B$-matrix, i.e. $b_{i_{k}, j_{k}}$. As we will show, this structure of the $\tau$-functions will be important to identify a soliton solution in the asymptotics $y \rightarrow \pm \infty$.

2.1. The $B$-matrix. Since the coefficients of the $\tau$-function are determined by the $B$-matrix, we give some remarks on the structure of the matrix. For a generic $4 N \times 4 N$ skew-symmetric matrix $B$, i.e. $B \in \mathfrak{s o}(4 N)$, there is a decomposition called skew-Borel decomposition [1],

$$
B=L J_{0} L^{T},
$$

where $L \in \mathcal{G}$, the group of invertible elements in the set of lower-triangular matrices with nonzero $2 \times 2$ blocks proportional to identity along the diagonal, i.e.

$$
\mathcal{G}:=\left\{\left(\begin{array}{cccccc}
a_{1} & 0 & \ldots & \ldots & 0 & 0 \\
0 & a_{1} & \ldots & \ldots & 0 & 0 \\
* & * & \ddots & \ddots & \vdots & \vdots \\
* & * & \ddots & \ddots & \vdots & \vdots \\
* & * & * & * & a_{2 N} & 0 \\
* & * & * & * & 0 & a_{2 N}
\end{array}\right) \mid \prod_{i=1}^{2 N} a_{i} \neq 0\right\}
$$

The matrix $J_{0}$ is the $4 N \times 4 N$ skew-symmetric matrix whose $2 \times 2$ diagonal blocks are given by $\left(\begin{array}{cc}0 & 1 \\ -1 & 0\end{array}\right)$ and all the other entries are zero, i.e.

$$
J_{0}:=\left(\begin{array}{cccccc}
0 & 1 & & & & 0 \\
-1 & 0 & & & & \\
& & \ddots & \ddots & & \\
& & \ddots & \ddots & & \\
& & & & 0 & 1 \\
0 & & & -1 & 0
\end{array}\right)
$$

A non-generic element of $\mathfrak{s o}(4 N)$ may be obtained by a permutation matrix $\pi \in S_{4 N}$ with $\pi^{-1}=\pi^{T}$ (i.e. $\pi \in O(4 N)$ ), that is, we have

$$
B=\pi L J_{0} L^{T} \pi^{-1}=(\pi L) J_{0}(\pi L)^{T},
$$

where $L$ is not a generic element in $\mathcal{G}$.

Example 2.2. Consider the case $N=1$ ( $4 \times 4 B$-matrix). Then the generic element $B$ can be expressed by $B=L J_{0} L^{T}$ with

Then the matrix $B$ is given by

$$
L=\left(\begin{array}{llll}
a & 0 & 0 & 0 \\
0 & a & 0 & 0 \\
b & d & f & 0 \\
c & e & 0 & f
\end{array}\right) \in \mathcal{G}
$$

$$
B=L J_{0} L^{T}=\left(\begin{array}{cccc}
0 & a^{2} & a d & a e \\
& 0 & -a b & -a c \\
& & 0 & -c d+b e+f^{2} \\
& & & 0
\end{array}\right) .
$$


(We leave the lower triangular part in blank for skew symmetric matrix.) If a skew-symmetric matrix has zero at $(1,2)$-entry $\left(\right.$ i.e. $\left.b_{1,2}=0\right)$ and others are nonzero, such matrix, say $B_{1}$, cannot be expressed in this form. In this case, one can consider the form $B=(\pi L) J_{0}(\pi L)^{T}$ with

$$
w=s_{2,3}:=\left(\begin{array}{cccc}
1 & 0 & 0 & 0 \\
0 & 0 & 1 & 0 \\
0 & 1 & 0 & 0 \\
0 & 0 & 0 & 1
\end{array}\right), \quad L=\left(\begin{array}{cccc}
a & 0 & 0 & 0 \\
0 & a & 0 & 0 \\
b & d & f & 0 \\
0 & e & 0 & f
\end{array}\right) .
$$

The matrix $B_{1}$ is then given by

$$
B_{1}=\left(s_{2,3} L\right) J_{0}\left(s_{2,3} L\right)^{T}=\left(\begin{array}{cccc}
0 & 0 & a^{2} & a e \\
& 0 & a b & b e+f^{2} \\
& & 0 & -a d \\
& & & 0
\end{array}\right) .
$$

In particular, with $L=I d$, the $4 \times 4$ identity matrix, we have $B_{1}=J_{1}$ defined by

$$
J_{1}:=\left(\begin{array}{cccc}
0 & 0 & 1 & 0 \\
& 0 & 0 & 1 \\
& & 0 & 0 \\
& & & 0
\end{array}\right) \text {. }
$$

Other non-generic element of $B$-matrix, say $B_{2}$, having $b_{1,2}=b_{1,3}=0$ can be expressed by $B_{2}=$ $\left(s_{2,4} L\right) J_{0}\left(s_{2,4} L\right)^{T}$,

$$
B_{2}=\left(\begin{array}{cccc}
0 & 0 & 0 & a^{2} \\
& 0 & f^{2} & a c \\
& & 0 & -a b \\
& & & 0
\end{array}\right)
$$

with

$$
s_{2,4}=\left(\begin{array}{cccc}
1 & 0 & 0 & 0 \\
0 & 0 & 0 & 1 \\
0 & 0 & -1 & 0 \\
0 & 1 & 0 & 0
\end{array}\right) \quad \text { and } \quad L=\left(\begin{array}{cccc}
a & 0 & 0 & 0 \\
0 & a & 0 & 0 \\
b & 0 & f & 0 \\
c & 0 & 0 & f
\end{array}\right) \text {. }
$$

In particular, for $L=I d$ we have $B_{2}=J_{2}$ defined by

$$
J_{2}:=\left(\begin{array}{cccc}
0 & 0 & 0 & 1 \\
& 0 & 1 & 0 \\
& & 0 & 0 \\
& & & 0
\end{array}\right)
$$

In the next section we show that those skew-symmetric matrices $J_{0}, J_{1}$ and $J_{2}$ define all the one D-soliton solutions.

In general, one can start with $J_{0}$ to define the $\tau$-functions, that is, $\tau_{n}=\operatorname{Pf}\left(\mathcal{Q}_{n}\right)$ with $\mathcal{Q}_{n}=\mathcal{E}_{n} J_{0} \mathcal{E}_{n}^{T}$ of (1.6). Then consider a generalization of the $\mathcal{Q}_{n}$-matrix by replacing $J_{0}$ with $J_{\pi}:=\pi J_{0} \pi^{-1}$ for some $\pi \in S_{4 N} \cap O(4 N)$. The role of $\pi$ can be understood as the change of order of the column vectors in $\mathcal{E}_{n}$, i.e.

$$
\mathcal{E}_{n} \pi:=\left(\mathbf{E}_{1}, \mathbf{E}_{2}, \ldots, \mathbf{E}_{4 N}\right) \pi=\left( \pm \mathbf{E}_{\pi(1)}, \pm \mathbf{E}_{\pi(2)}, \ldots, \pm \mathbf{E}_{\pi(4 N)}\right)
$$

where the column vector $\mathbf{E}_{k}:=\left(E_{k}, E_{k}^{(1)}, \ldots, E_{k}^{(2 n-1)}\right)^{T}$, and $\pi(k)$ indicates the permutation $k \rightarrow$ $\pi(k)$. The signs in $w$ should be chosen properly so that the new $\tau$-function $\tau_{n}^{\prime}=\left(\mathcal{E}_{n} \pi\right) J_{0}\left(\mathcal{E}_{n} \pi\right)^{T}$ is sign-definite, i.e. non-singular (see Section 4 for more details).

\section{One D-soliton And two A-solitons}

Here we discuss the cases with $4 \times 4$ skew-symmetric matrix $B$, and show that the cases include one D-soliton and two A-solitons. 
3.1. D-solitons. In Introduction, we show an example of one D-soliton (1.8) whose $\tau$-function is given by $\tau_{1}=\operatorname{Pf}\left(\mathcal{E}_{1} J_{0} \mathcal{E}_{1}^{T}\right)$ with $J_{0}$ in $(2.1)$ and $\mathcal{E}_{1}$ in Lemma 2.1 for $N=1$. This D-soliton is denoted as $[1,2: 3,4]$, since it exchanges the asymptotic values of $w=\left(\ln \tau_{1}\right)_{x}$ as $(1,2) \leftrightarrow(3,4)$ (recall $\left.(i, j):=p_{i}+p_{j}\right)$. A general form of the one D-soliton can be expressed as $\left[i_{1}, j_{1}: i_{2}, j_{2}\right]$ where $i_{1}<i_{2}$ and $i_{k}<j_{k}$. There are three cases,

$$
[1,2: 3,4], \quad[1,3: 2,4], \quad[1,4: 2,3],
$$

which correspond to the D-solitons generated respectively by the skew-symmetric matrices, $J_{0}, J_{1}$ and $J_{2}$ in Example 2.2 as the $B$-matrices. Recall here that the soliton label $[i, j: k, l]$ indicates the nonzero elements $b_{i, j}$ and $b_{k, l}$ in the $B$-matrix. Namely for the $J_{k}$-matrix with nonzero entries with 1 at $\left(i_{1}, j_{1}\right)$ and $\left(i_{2}, j_{2}\right)$ in the upper-triangular part, the $\tau$-function is given by

$$
\tau_{1}=Q_{1,2}=\left(p_{j_{1}}-p_{i_{1}}\right) E_{i_{1}} E_{j_{1}}+\left(p_{j_{2}}-p_{i_{2}}\right) E_{i_{2}} E_{j_{2}} .
$$

Then the function $w=\left(\ln \tau_{1}\right)_{x}$ is

$$
w(x, y, t):=\frac{\partial}{\partial x} \ln \tau_{1}=\frac{1}{2} \sum_{k=1}^{4} p_{k}+\frac{1}{2}\left(p_{i_{2}}+p_{j_{2}}-p_{i_{1}}-p_{j_{1}}\right) \tanh \frac{1}{2}\left(\theta_{i_{2}, j_{2}}-\theta_{i_{1}, j_{1}}\right)
$$

with $\theta_{i, j}=\theta_{i}+\theta_{j}+\ln \left|p_{i}-p_{j}\right|$. For each asymptotic of $x \rightarrow \pm \infty$, one of the exponential terms in the $\tau$-function (3.1) becomes dominant, and we have

$$
w(x, y, t) \rightarrow \begin{cases}p_{i_{1}}+p_{j_{1}}, & \text { as } x \rightarrow-\infty \\ p_{i_{2}}+p_{j_{2}}, & \text { as } x \rightarrow \infty\end{cases}
$$

Thus one D-soliton exchanges $\left(i_{1}, j_{1}\right) \leftrightarrow\left(i_{2}, j_{2}\right)$ with the values of $w$, and is denoted by $\left[i_{1}, j_{1}: i_{2}, j_{2}\right]$, as an element of the Weyl group of D-type.

One soliton solution $u=2 w_{x}$ is a plane wave having the form,

$$
u(x, y, t)=\phi\left(k_{x} x+k_{y} y-\omega t\right),
$$

Let us denote the wavenumber $\mathbf{k}=\left(k_{x}, k_{y}\right)$ and the frequency $\omega$ for $(3.2)$ by $\mathbf{k}\left[i_{1}, j_{1}: i_{2}, j_{2}\right]$ and $\omega\left[i_{1}, j_{1}: i_{2}, j_{2}\right]$, i.e.

$$
\left\{\begin{array}{l}
\mathbf{k}\left[i_{1}, j_{1}: i_{2}, j_{2}\right]=\left(p_{i_{2}}+p_{j_{2}}-p_{i_{1}}-p_{j_{1}}, p_{i_{2}}^{2}+p_{j_{2}}^{2}-p_{i_{1}}^{2}-p_{j_{1}}^{2}\right) \\
\omega\left[i_{1}, j_{1}: i_{2}, j_{2}\right]=-\left(p_{i_{2}}^{3}+p_{j_{2}}^{3}-p_{i_{1}}^{3}-p_{j_{1}}^{3}\right)
\end{array}\right.
$$

The slope (or velocity) of the soliton in the $x-y$ plane is given by $c:=d x / d y=-k_{y} / k_{x}=-\left(p_{i_{2}}^{2}+\right.$ $\left.p_{j_{2}}^{2}-p_{i_{1}}^{2}-p_{j_{1}}^{2}\right) /\left(p_{i_{2}}+p_{j_{2}}-p_{i_{1}}-p_{j_{1}}\right)$. The peak of the soliton then determines the line in the $x-y$ plane given by the equation $\theta_{i_{1}, j_{1}}(x, y, t)=\theta_{i_{2}, j_{2}}(x, y, t)$ for each $t$.

We also note that the velocity $c$ of one D-soliton can be obtained as follows: First note that a D-soliton of $[i, j: k, l]$ gives a line $\theta_{i, j}=\theta_{k, l}$. Then setting $x=c y$ for this equation, we have $\theta_{i, j}=u_{i, j}(c) y+\theta_{i, j}^{0}$ with

$$
u_{i, j}(c):=\left(p_{i}+p_{j}\right) c+p_{i}^{2}+p_{j}^{2} .
$$

Then taking the limit $|y| \rightarrow \infty$ for the equation $\theta_{i, j}=\theta_{k, l}$, the velocity $c$ of the D-soliton is determined by $u_{i, j}(c)=u_{k, l}(c)$, i.e. the intersection point of $u_{i, j}$ and $u_{k, l}$. In Figure 1 , we show $u_{i, j}(c)$ of $(3.3)$ for the parameter $\mathbf{p}:=\left(p_{1}, \ldots, p_{4}\right)=(-2,-1,0,3)$. Each solid dot gives the velocity of one D-soliton, and each open circle gives the velocity of an A-soliton, e.g. the point at $u_{1,2}=u_{1,3}$ gives the velocity of $[2: 3]$-soliton. This implies that if $b_{1,2}$ and $b_{1,3}$ are only nonzero elements in the $B$-matrix, then $\tau_{1}$ gives one A-soliton of [2:3], i.e. $\tau_{1}=\left(b_{1,2}\left(p_{2}-p_{1}\right) E_{2}+b_{1,3}\left(p_{3}-p_{1}\right) E_{3}\right) E_{1}$. Figure 1 can be also used to determine the solitons appearing from the $\tau_{1}$-function in the asymptotics $|y| \rightarrow \infty$. For example, if $b_{i, j}>0$ for all $1 \leq i<j \leq 4$, then $\tau_{1}$ contains all six terms $E_{i} E_{j}$ with $i<j$. Then from Figure 1, we have two A-soliton solutions of T-type, [1:3] and [2:4] (see below for the details). 


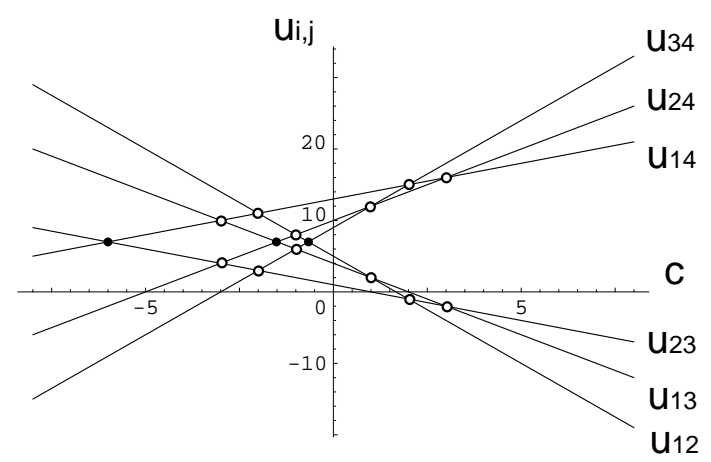

Figure 1 . The functions $u_{i, j}(c)$ and the velocities of solitons. The intersection point between $u_{i, j}$ and $u_{k, l}$ gives the velocity of D-soliton $[i, j: k, l]$. The solid dots give the velocities of D-solitons, and the open circles give the velocities of Asolitons, e.g. the velocity of $[1: 2]$-soliton is $c=-\left(p_{1}+p_{2}\right)$ which is given by either $u_{1,4}=u_{2,4}$ or $u_{1,3}=u_{2,3}$.
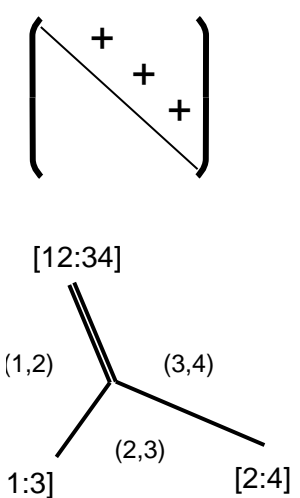
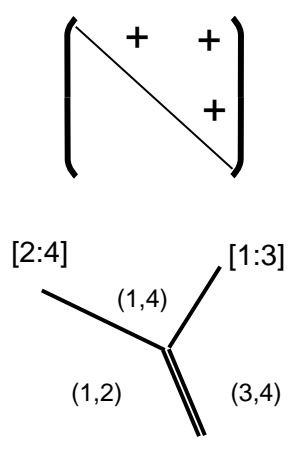

[12:34]
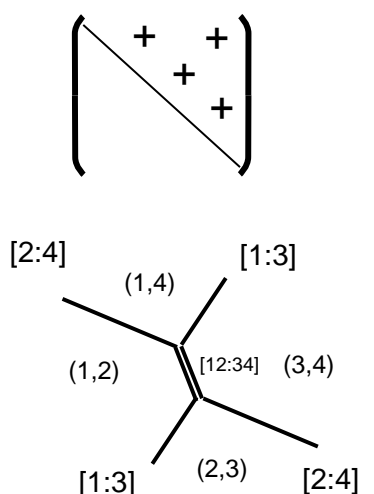

Figure 2. Resonant bifurcations of the D-soliton [1,2:3,4]. Each region, $(i, j)$, corresponds to a dominant exponential marked by the element $b_{i, j}$. For example, with $b_{2,3} \neq 0,[1,2: 3,4]$-D-soliton bifurcates into [1:3] and [2:4] A-solitons as $y \rightarrow-\infty$ (top figure). Those solitons can be found from Figure 1 .

3.2. Two A-solitons. One D-soliton can be considered as a degenerate case of two A-solitons (recall that A-soliton exchanges single letter as $[i: j]$, while D-soliton exchanges two letters as $[i, j: k, l])$. Namely, D-soliton of $[i, j: k, l]$ can be considered as the "product" of two A-solitons of $[i: k]$ and $[j: l]$ or $[i: l]$ and $[j: k]$, i.e. $[i, j: k, l]=[i: k] \cdot[j: l]=[i: l] \cdot[j: k]$ as the product of two permutations. Then two A-soliton solutions can be obtained by adding extra exponential terms, i.e. extra nonzero entries to the $B$-matrix. If one adds just one term, then we have Y-shaped solution satisfying the resonant condition. For example, for the $B$-matrix having two nonzero entries $b_{i_{1}, j_{1}}$ and $b_{i_{2}, j_{2}}$ in the upper triangular part, if we add a nonzero entry at either $\left(i_{1}, j_{2}\right)$ or $\left(i_{2}, j_{2}\right)$, we obtain a resonant $\mathrm{Y}$-shaped soliton with the resonant condition,

$$
\left\{\begin{array}{l}
\mathbf{k}\left[i_{1}: i_{2}\right]+\mathbf{k}\left[j_{1}: j_{2}\right]=\mathbf{k}\left[i_{1}, j_{1}: i_{2}, j_{2}\right] \\
\omega\left[i_{1}: i_{2}\right]+\omega\left[j_{1}: j_{2}\right]=\omega\left[i_{1}, j_{1}: i_{2}, j_{2}\right] .
\end{array}\right.
$$

Here the wavenumber $\mathbf{k}[i: j]$ and the frequency $\omega[i: j]$ are for the one soliton solution of the KP equation, i.e.

$$
\mathbf{k}\left[i_{1}: i_{2}\right]=\left(p_{i_{1}}-p_{i_{2}}, p_{i_{1}}^{2}-p_{i_{2}}^{2}\right), \quad \omega\left[i_{1}: i_{2}\right]=p_{i_{1}}^{3}-p_{i_{2}}^{3},
$$

In Figure 2 (see also Example 3.1 below), we show the resonant bifurcation of $[1,2: 3,4]$-soliton 
into two A-solitons of $[1: 3]$ and [2:4]. Those solitons can be found from Figure 1. For example, in the left figure of Figure 2, we have $u_{1,2}, u_{2,3}$ and $u_{3,4}$ from the nonzero entries of the $B$-matrix. Then the three intersection points of the graphs of those $u_{i, j}$ in Figure 1 give one D-soliton (solid dot) and two A-solitons (open circles). Figure 1 also shows that the solid dot indicates the pair of dominant exponentials $\left(E_{1,2}, E_{3,4}\right)$ for $[1,2: 3,4]$-soliton as $y \rightarrow \infty$, while the open circles show the dominant pairs $\left(E_{1,2}, E_{2,3}\right)$ for $[1: 3]$-soliton and $\left(E_{2,3}, E_{3,4}\right)$ for $[2: 4]$-soliton as $y \rightarrow-\infty$.

Since there are at least four disconnected regions in the $x-y$ plane divided by two line solitons, one needs to add at least two extra nonzero (positive) entries. Figure 2 shows the resonant bifurcations of a D-soliton into two A-solitons. Two soliton solution in this figure is a new T-type (referred to as TD-type) which does not exist in the KP equation (i.e. $v^{ \pm} \neq 0$ ). The T-type of the KP equation can be obtained by a $B$-matrix with $\operatorname{Det}(B)=0$, e.g.

$$
B=\left(\begin{array}{llll}
0 & 1 & 2 & 1 \\
& 0 & 1 & 1 \\
& & 0 & 1 \\
& & & 0
\end{array}\right)
$$

Note $\tau_{2}=0$ with this matrix due to $\operatorname{Pf}(B)=0$. One should also note that the generic skewsymmetric $B$-matrix having all nonzero entries gives a two A-soliton solution of T-type (having a resonant hole), but it is not a solution of the KP equation. In this case, since those solitons are A-solitons, the function $v^{+} v^{-}=\tau_{2} / \tau_{1}^{2}$ appears only locally in the $x$ - $y$ plane. Both $\tau$-functions for T-type two-soliton solutions of KP and DKP equations have six independent exponentials, i.e. $E_{i, j}$ for $1 \leq i<j \leq 4$, which is the key to produce T-type resonant interaction of two A-solitons (see also $[2,8])$.

Example 3.1. Consider $[1,2: 3,4]$-D-soliton which corresponds to the $B$-matrix having nonzero entries at $(1,2)$ and $(3,4)$, i.e. $B=J_{0}$ in $(2.1)$ :

(a) $[1: 3]$ - and [2:4]-A-solitons (TD-type) by putting +1 at the entries $(1,4)$ and $(2,3)$.

(b) $[1: 4]$ - and $[2: 3]$-A-solitons (P-type) by putting +1 at the entries $(1,3)$ and $(2,4)$.

Note that the TD-type in the case a) is not the same as the one of the KP-solitons, i.e. there is no resonance in this case. Original T-type of two A-solitons is obtained by the $B$-matrix having all nonzero entries and $\operatorname{Det}(B)=0$. Again there are three types of two A-solitons for DKP. These types play an important role for the classification problem as a building block. Figure 3 shows the diagrams of the three fundamental types of two A-solitons. The middle diagrams of 8-gon connect the types with the corresponding $B$-matrices as follows: We have nonzero $b_{i, j}$ if the pair $(i, j)$ is connected in the diagram, and a corresponding A-soliton of $[k: l]$ is given by the pair $(k, l)$ having no connection in the diagram. Also note that the corresponding $B$-matrix for O-type is not in the generic one given in (2.2), but in (2.3) (compare this with the O-type of KP solitons which belong to a Schubert cell of codimension one [8]). Figure 4 shows those types of two A-solitons, which are the same as 2-soliton solutions of the KP equation (note here that we have set $\tau_{2}=0$ for all the types, i.e. $\tau_{1}$ gives a solution of the KP equation). The parameters are chosen as $\mathbf{p}=(-2,-1,0,3)$, and the velocities of solitons can be found from Figure 1.

\section{Solitons Generated By $8 \times 8 B$-MAtrices}

Since each D-soliton has four phases, we need eight phases to describe a two D-soliton solution. The $\tau_{2}$-function in the case with $8 \times 8 B$-matrix is given by

$$
\tau_{2}=\sum_{1 \leq i_{1}<\cdots<i_{4} \leq 8} \operatorname{Pf}\left(B\left(i_{1}, \ldots, i_{4}\right)\right) \Delta\left(i_{1}, \ldots, i_{4}\right) \exp \left(\sum_{k=1}^{4} \theta_{i_{k}}\right) .
$$

where $\Delta\left(i_{1}, \ldots, i_{4}\right)=\prod_{1 \leq j<k \leq 4}\left(p_{i_{j}}-p_{i_{k}}\right)>0$, and the pfaffian is calculated as

$$
\operatorname{Pf}\left(B\left(i_{1}, \ldots, i_{4}\right)\right)=b_{i_{1}, i_{2}} b_{i_{3}, i_{4}}-b_{i_{1}, i_{3}} b_{i_{2}, i_{4}}+b_{i_{1}, i_{4}} b_{i_{2}, i_{3}} .
$$



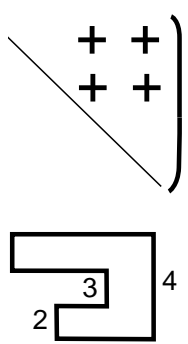

$\multimap 2$

$30-04$

O-type
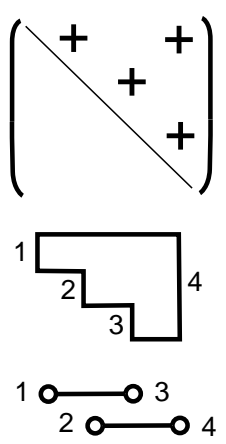

TD-type
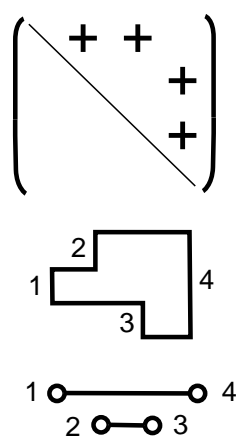

P-type

Figure 3. Three fundamental types of two A-solitons obtained from D-soliton. Each diagram provides the relation between $B$-matrix and the corresponding twosoliton solution, i.e. $b_{i, j} \neq 0$ if and only if the pair $(i, j)$ is directly connected in the middle diagram, and $[k: l]$-soliton implies that the pair $(k, l)$ has no direct connection. For example, in O-type, two A-solitons are labeled [1:2] and [3:4], then we have the middle diagram to show the connections between the numbers in the pairs, i.e. $(1,2)$ and $(3,4)$ are not connected. This diagram implies we have nonzero elements $b_{1,3}, b_{1,4}, b_{2,3}$ and $b_{2,4}$.

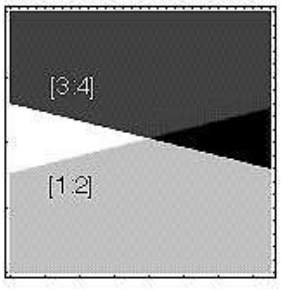

O-type

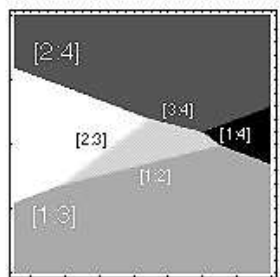

T-type

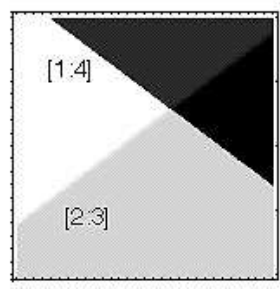

P-type

Figure 4. Three fundamental types of two A-solitons. Those solutions have the same topological feature as the two soliton solutions of the KP equation (see also Figure 3 in [8]). The difference between those solutions appears as the existence of localized solutions $v^{ \pm}$(if $v^{+} v^{-}=0$, then the DKP solution is also the KP solution). However note that the TD-type in Figure 3 does not have a resonant quadrangle. The figures show the counter plots of the function $w=(\ln \tau)_{x}$ in the $x$-y plane for a fixed $t$, and the contrast of the shades shows the different values of $w$ which increases in the positive $x$-direction.

Let us now construct the $B$-matrices for two D-soliton solutions which consist of only four nonzero entries in the upper triangular parts. We follow the steps:

(1) Take four pairs $\left(i_{k}, j_{k}\right), k=1, . ., 4$ with $i_{k}<j_{k}$ from the set $\{1, \ldots, 8\}$, such that

$$
1=i_{1}<i_{2}<\cdots<i_{4}, \quad j_{k} \neq j_{l},(k \neq l) .
$$

There are $(2 \times 4-1) ! !=105$ ways to make those pairs (compare this with $\left(\mathbf{n}^{-}, \mathbf{n}^{+}\right)$in the case of 4 A-solitons [8]). Those four pairs give four nonzero elements in the $B$-matrix, i.e. $\left(i_{k}, j_{k}\right) \equiv b_{i_{k}, j_{k}}$ for $k=1, \ldots, 4$.

(2) Determine the signs of each pairs $\left(i_{k}, j_{k}\right)$, so that all the pfaffians in the expansion of $\tau_{2}$ associated with the $B$-matrix have the same sign. Since the pairs $\left(i_{k}, j_{k}\right)$ are all different, each pfaffian has just one term, denoted by $\operatorname{Pf}((i, j),(k, l))= \pm b_{i, j} b_{k, l}$. Here the sign depends 
on the pairs, for examples,

$$
\operatorname{Pf}((1,3),(5,7))=b_{1,3} b_{5,7}, \quad \operatorname{Pf}((2,6),(4,8))=-b_{2,6} b_{4,8} .
$$

In order to determine the sign of the pfaffian $\operatorname{Pf}((i, j),(k, l))= \pm b_{i, j} b_{k, l}$, we define:

Definition 4.1. We say that the pairs $\left(i_{j}, i_{k}\right)$ and $\left(i_{l}, i_{m}\right)$ with $i_{j}<i_{l}$ have a partial overlap, if $i_{j}<i_{l}<i_{k}<i_{m}$. Otherwise, we say that the pairs have a non-partial overlap (i.e. total or no overlap). Let us introduce the sign of overlap between $\left(i_{k}, j_{k}\right)$ and $\left(i_{l}, j_{l}\right)$,

$$
\sigma_{k l}= \begin{cases}- & \text { if } i_{k}<i_{l}<j_{k}<j_{l} \text { (partial overlap) } \\ + & \text { otherwise }\end{cases}
$$

Then we have $\operatorname{Pf}\left(\left(i_{k}, j_{k}\right),\left(i_{l}, j_{l}\right)\right)=\sigma_{k l} b_{i_{k}, j_{k}} b_{i_{l}, j_{l}}$.

Now we have:

Lemma 4.1. Suppose that the signs $\sigma_{k l}$ satisfiy

$$
\sigma_{12} \sigma_{13} \sigma_{14}=\sigma_{12} \sigma_{23} \sigma_{24}=\sigma_{13} \sigma_{23} \sigma_{34}=\sigma_{14} \sigma_{24} \sigma_{34} .
$$

Then one can make the $\tau$-function $\tau_{2}$ to be sign-definite, that is, all $\operatorname{Pf}\left(B\left(i_{1}, \ldots, i_{4}\right)\right)$ take the same sign.

Proof. We denote $\epsilon_{k}=\operatorname{sgn}\left(i_{k}, j_{k}\right)$, so that $\operatorname{sgn}\left(\operatorname{Pf}\left(\left(i_{k}, j_{k}\right)\left(i_{l}, j_{l}\right)\right)\right)=\sigma_{k l} \epsilon_{k} \epsilon_{l}$. The condition that all the terms have the same sign requires

$$
\sigma_{k l} \epsilon_{k} \epsilon_{l}=\sigma_{k^{\prime} l^{\prime}} \epsilon_{k^{\prime}} \epsilon_{l^{\prime}}
$$

for any $k, l$ and $k^{\prime}, l^{\prime}$. This leads to $\sigma_{12} \sigma_{34}=\sigma_{13} \sigma_{24}=\sigma_{14} \sigma_{23}$ which gives the assertion.

Applying the Lemma, one can prove:

Proposition 4.1. Let $\mathbf{i}=\left(i_{1}, \ldots, i_{4}\right)$ and $\mathbf{j}=\left(j_{1}, \ldots, j_{4}\right)$. Then there exist 33 cases of sign-definite $\tau$-functions whose $B$-matrices have nonzero entries only at $\left(i_{k}, j_{k}\right)$ and $\left(j_{k}, i_{k}\right)$ for $k=1, \ldots, 4$. Those cases are given by:

(a) For $\mathbf{i}=(1,2,3,4)$, there are 8 cases with $\mathbf{j}=\left(j_{1}, \ldots, j_{4}\right)$;

$(5,6,7,8),(5,8,7,6),(6,5,8,7),(6,7,8,5)$

$(7,8,5,6),(7,6,5,8),(8,5,6,7),(8,7,6,5)$.

(b) For $\mathbf{i}=(1,2,3,5)$, there are 4 cases,

$$
(4,7,6,8),(4,7,8,6),(6,7,4,8),(8,7,4,6) .
$$

(c) For $\mathbf{i}=(1,2,3,6)$, there are 4 cases,

$$
(4,5,8,7),(5,4,7,8),(7,4,5,8),(8,5,4,7) .
$$

(d) For $\mathbf{i}=(1,2,4,5)$, there are 4 cases,

$$
(3,8,6,7),(3,6,8,7),(6,3,7,8),(8,3,7,6) .
$$

(e) For $\mathbf{i}=(1,2,4,6)$, there is one case, $(8,3,5,7)$.

(f) For $\mathbf{i}=(1,2,3,7)$, there are two cases, $(4,5,6,8),(6,5,4,8)$.

(g) For $\mathbf{i}=(1,3,4,5)$, there are two cases, $(2,6,7,8),(2,8,7,6)$.

(h) For $\mathbf{i}=(1,2,4,7)$, there is one case, $(6,3,5,8)$.

(i) For $\mathbf{i}=(1,3,4,6)$, there $i s$ one case, $(2,8,5,7)$.

(j) For $\mathbf{i}=(1,2,5,6)$, there are two cases, $(3,4,7,8),(4,3,8,7)$.

(k) For $\mathbf{i}=(1,2,5,7)$, there is one case, $(4,3,6,8)$.

(l) For $\mathbf{i}=(1,3,5,6)$, there is one case, $(2,4,8,7)$.

(m) For $\mathbf{i}=(1,3,4,7)$, there is one case, $(2,6,5,8)$.

(n) For $\mathbf{i}=(1,3,5,7)$, there is one case, $(2,4,6,8)$.

The case (n) corresponds to the $B$-matrix $B=J_{0}$ defined in (2.1), and the $B$-matrices for other cases can be expressed as $B=J_{\pi}:=\pi J_{0} \pi^{T}$ for some $\pi \in S_{8}$ with appropriate signs in the entries of $\pi$. Those signs are determined so that the $\tau$-function is non-singular. 
4.1. Two and three D-solitons. We now classify D-solitons given by the $\tau$-functions associated with the $B$-matrices obtained in Proposition 4.1. We obtain:

Theorem 4.2. Suppose we have the order, $\left(i_{1}, j_{1}\right)<\left(i_{2}, j_{2}\right)<\left(i_{3}, j_{3}\right)<\left(i_{4}, j_{4}\right)$ with $\left(i_{k}, j_{k}\right):=$ $p_{i_{k}}+p_{j_{k}}$. Then as a generic situation, the D-soliton solutions of the DKP equation turns out to be the following two cases;

(i) two $D$-solitons of $\left[i_{1}, j_{1}: i_{3}, j_{3}\right],\left[i_{2}, j_{2}: i_{4}, j_{4}\right]$ or $\left[i_{1}, j_{1}: i_{4}, j_{4}\right],\left[i_{2}, j_{2}: i_{3}, j_{3}\right]$,

(ii) three D-solitons of $\left[i_{k}, j_{k}: i_{\alpha}, j_{\alpha}\right],\left[i_{l}, j_{l}: i_{\alpha}, j_{\alpha}\right]$ and $\left[i_{m}, j_{m}: i_{\alpha}, j_{\alpha}\right]$ for $\alpha=2$ or $3(\alpha, k, l, m$ are all distinct).

Proof. First we note that each $\tau_{2}$-function associated with the $B$-matrix given in Proposition 4.1 has six exponential terms. We denote them as $\hat{E}_{k} \hat{E}_{l}$ for $1 \leq k<l \leq 4$, where $\hat{E}_{k}:=E_{i_{k}, j_{k}}=$ $\exp \left(\left(p_{i_{k}}+p_{j_{k}}\right) x+\left(p_{i_{k}}^{2}+p_{j_{k}}^{2}\right) y+\theta_{k}^{0}\right)$ for a fixed $t$. To identify two dominant exponents which determine an asymptotic D-soliton, we set $x=c y$ and define $u_{i, j}:=\left(p_{i}+p_{j}\right) c+p_{i}^{2}+p_{j}^{2}$ (see Section 3 ). Then for large $|y|$, the velocity $c$ of asymptotic soliton can be given by the intersection point of $u_{i_{k}, j_{k}}=u_{i_{l}, j_{l}}$, where as a generic situation only two exponential terms become dominant, say $\hat{E}_{k} \hat{E}_{m}$ and $\hat{E}_{l} \hat{E}_{m}$ (see also [2] for the details, and Figure 5 below). The soliton is then identified as $\left[i_{k}, j_{k}: i_{l}, j_{l}\right]$. Then there are two cases where the number of solitons, i.e. the number of balancing pairs of dominant exponentials, is either two or three. To show this, we consider, for example, the case with $\mathbf{i}=(1,2,3,4), \mathbf{j}=(5,6,7,8)$. We set the parameter $\mathbf{p}$ so that $p_{1}^{(1)}<p_{2}^{(1)}<p_{3}^{(1)}<p_{4}^{(1)}$ whith $p_{k}^{(1)}:=p_{i_{k}}+p_{j_{k}}$. Keeping this order, one can choose $\mathbf{p}$, so that we have $p_{4}^{(2)}<p_{3}^{(2)}<p_{2}^{(2)}<p_{1}^{(2)}$ with $p_{k}^{(2)}:=p_{i_{k}}^{2}+p_{j_{k}}^{2}$. (This can be done with a large negative value for $p_{1}$ and a small positive $p_{8}$.) Then it is easy to see from the graph of $u_{k}:=u_{i_{k}, j_{k}}$ that one can have either two D-soliton of $\left[i_{1}, j_{1}: i_{3}, j_{3}\right]$ and $\left[i_{2}, j_{2}: i_{4}, j_{4}\right]$ or three D-soliton of $\left[i_{1}, j_{1}: i_{3}, j_{3}\right],\left[i_{2}, j_{2}: i_{3}, j_{3}\right]$ and $\left[i_{3}, j_{3}: i_{4}, j_{4}\right]$. The parameters $\mathbf{p}=\left(p_{1}, \ldots, p_{8}\right)$ can take the same values except $p_{8}$ for both cases (Figure 5 gives an example for this, also see below).

One can also make a different order for $p_{k}^{(2)}$, e.g. $p_{3}^{(2)}<p_{2}^{(2)}<p_{1}^{(2)}<p_{4}^{(2)}$ (by taking large positive $\left.p_{8}\right)$. Then one can have three D-soliton of $\left[i_{1}, j_{1}: i_{2}, j_{2}\right],\left[i_{2}, j_{2}: i_{3}, j_{3}\right]$ and $\left[i_{2}, j_{2}: i_{4}, j_{4}\right]$ with the velocities $c_{2,4}<c_{1,2}<c_{2,3}$ where $c_{k, l}$ is the velocity of $\left[i_{k}, j_{k}: i_{l}, j_{l}\right]$-soliton (recall that those are given by the intersection $u_{k}=u_{l}$ with $u_{k}=u_{i_{k}, j_{k}}$ ).

Thus there is a freedom in the ordering of $p_{k}^{(2)}$, and this is a key to generate three D-solitons (compare with the case for the KP equation $[2,8]$ where we have only two A-solitons for six exponential terms in the $\tau$-function).

In Figure 5, we illustrate the case of $\mathbf{i}=(1,2,3,5)$ and $\mathbf{j}=(4,7,6,8)$ : Here the ordering is $(1,4)<(2,7)<(3,6)<(5,8)$. Then depending on the parameters $p_{k}$ 's, the number of asymptotic D-solitons is either two or three. The left (right) figure shows the case of two (three) D-solitons, i.e. there are two (three) values of $c$ where two exponential terms become dominant. It is obvious that there is no case other than those two in the general case.

We note here that all cases in Proposition 4.1 have two-D-soliton solutions, but only some cases can have three-D-soliton solution. In particular, we have:

Proposition 4.2. The case (n) in Proposition 4.1 can have only two-D-soliton solutions of $[1,2$ : $5,6]$ and $[3,4: 7,8]$.

In order to prove the Proposition, we prepare the following Lemma: Let us first denote $u_{i_{k}, j_{k}}$ as $u_{k}$ as before:

$$
u_{k}(c):=\left(p_{i_{k}}+p_{j_{k}}\right) c+p_{i_{k}}^{2}+p_{j_{k}}^{2}, \quad k=1, \ldots, 4,
$$

where $\left(i_{k}, j_{k}\right)=(2 k-1,2 k)$ for the $B$-matrix of the case (n), i.e. $B=J_{0}$. Then we have:

Lemma 4.2. Let $c_{j, k}$ be the point of intersection given by $u_{j}(c)=u_{k}(c)$. Then for a fixed index $\alpha \in\{1, \ldots, 4\}$, we have

$$
c_{\alpha, k}<c_{\alpha, j}<c_{\alpha, i}, \quad \text { for } \quad i<j<k, \alpha \neq i, j, k .
$$



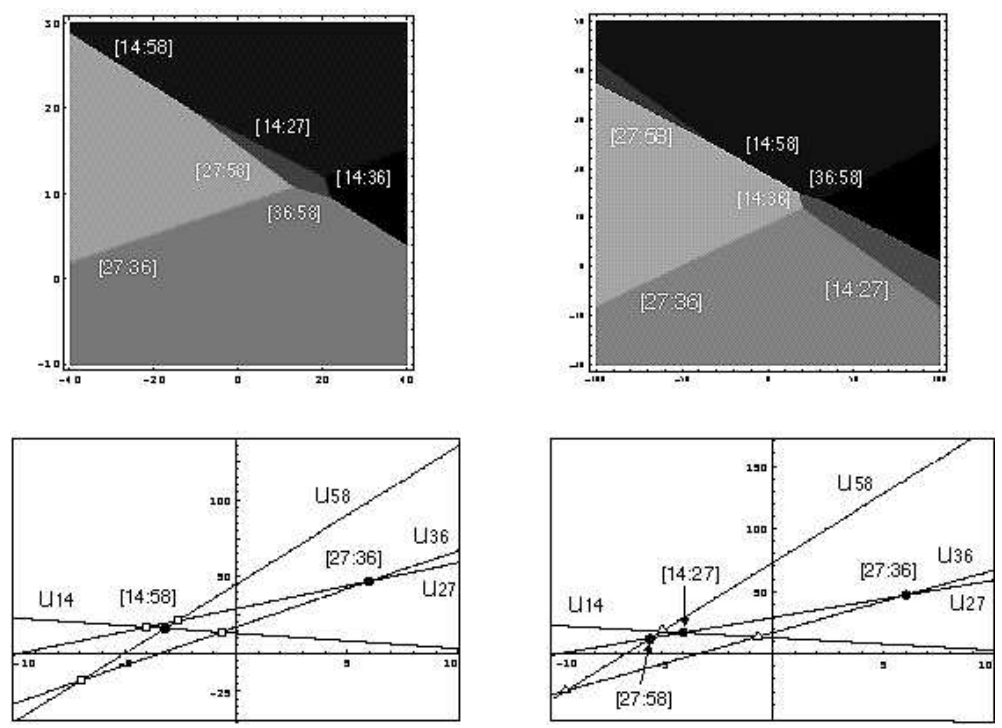

Figure 5. Two and three D-solitons associated with $\mathbf{i}=(1,2,3,5)$ and $\mathbf{j}=$ $(4,7,6,8)$. The left figure shows two D-solitons with $\mathbf{p}=(-3,-2,1,2,3,4,5,6)$. The right one shows three D-solitons with the same parameters except $p_{8}=8$. The bottom figures show the graphs of $u_{i, j}(c)=\left(p_{i}+p_{j}\right) c+p_{i}^{2}+p_{j}^{2}$. The dots in the graphs show the dominant two exponential terms, which determine the asymptotic D-solitons. The squares correspond to the intermediate D-solitons forming the resonant quadrangle, and the triangles correspond to the resonant intermediate solitons from the pairs of three asymptotic solitons as shown in the top figures.

Proof. From $u_{k}(c)=u_{j}(c)$, we have

$$
c_{j, k}=-\frac{\left(p_{2 j-1}^{2}+p_{2 j}^{2}\right)-\left(p_{2 k-1}^{2}+p_{2 k}^{2}\right)}{\left(p_{2 j-1}+p_{2 j}\right)-\left(p_{2 k-1}+p_{2 k}\right)} .
$$

Let us write $p_{2 k-1}$ and $p_{2 k}$ as

$$
p_{2 k-1}=P_{k}-\Delta_{k}, \quad p_{2 k}=P_{k}+\Delta_{k},
$$

that is, $P_{k}=\frac{1}{2}\left(p_{2 k-1}+p_{2 k}\right)$, and we have

$$
c_{j, k}=-\left(P_{j}+P_{k}\right)-\frac{\Delta_{j}^{2}-\Delta_{k}^{2}}{P_{j}-P_{k}} .
$$

Suppose $\alpha<j<k$ (other cases follow the similar argument). Then we have

$$
c_{\alpha, j}-c_{\alpha, k}=P_{k}-P_{j}+\frac{\Delta_{\alpha}^{2}-\Delta_{k}^{2}}{P_{\alpha}-P_{k}}-\frac{\Delta_{\alpha}^{2}-\Delta_{j}^{2}}{P_{\alpha}-P_{j}} .
$$

It is then easy to show $c_{\alpha, j}-c_{\alpha, k}>0 . \quad \square$

Now we give a proof of Proposition 4.2:

Proof. From Lemma 4.2, we have the order $c_{1,2}<c_{1,3}<c_{1,4}$. Also note that the slope of $u_{k}(c)$ is given by $p_{2 k-1}+p_{2 k}=: p_{k}^{(1)}$, hence $p_{1}^{(1)}<p_{2}^{(1)}<p_{3}^{(1)}<p_{4}^{(1)}$. Then we obtain qualitative graphs of $u_{k}$ as shown in Figure 6, and from the graphs, we can find the dominant pairs of the exponential terms in $\tau_{2}$ for $y \rightarrow \pm \infty$. This shows that the dominant pairs are given by $\left(\hat{E}_{1} \hat{E}_{4}, \hat{E}_{3} \hat{E}_{4}\right)$ and $\left(\hat{E}_{1} \hat{E}_{4}, \hat{E}_{1} \hat{E}_{2}\right)$ for $y \rightarrow \infty$, and $\left(\hat{E}_{1} \hat{E}_{2}, \hat{E}_{2} \hat{E}_{3}\right)$ and $\left(\hat{E}_{2} \hat{E}_{3}, \hat{E}_{3} \hat{E}_{4}\right)$ for $y \rightarrow-\infty$. This implies that we have two D-solitons of $[1,2: 5,6]$ and $[3,4: 7,8]$ (recall $\hat{E}_{k}=E_{2 k-1,2 k}$ ). This completes the proof. $\square$

We also have: 


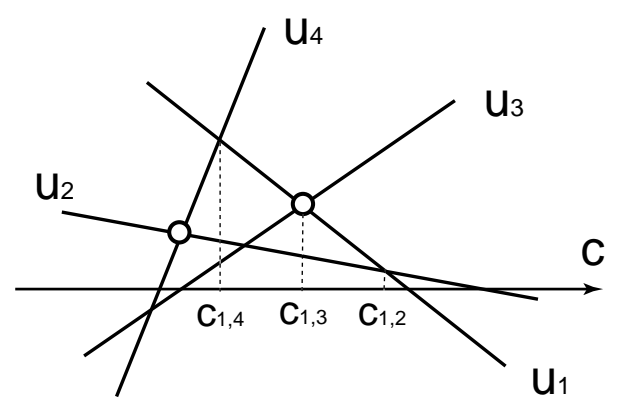

Figure 6 . The qualitative graphs of the functions $u_{k}(c)=\left(p_{2 k-1}+p_{2 k}\right) c+p_{2 k-1}^{2}+$ $p_{2 k}^{2}$ for the case (n) in Proposition 4.1 which states, e.g. $c_{1,4}<c_{1,3}<c_{1,2}$. The open circles correspond to two D-solitons of $[1,2: 5,6]$ with the velocity $c_{1,3}$ and $[3,4: 7,8]$ with $c_{2,4}$.

Proposition 4.3. The case with $\mathbf{i}=(1,2,3,4)$ and $\mathbf{j}=(8,7,6,5)$ (one of the cases in (a) of Proposition 4.1) can have both two and three D-solitons.

Proof. Follow the argument in the proof of Theorem 4.2. Also see the following examples:

(i) For two D-solitons, we take $\mathbf{p}:=\left(p_{1}, p_{2}, \ldots, p_{8}\right)=(-5,-4,-2,-1,0,3,3.5,7)$. Then the open circles in the left figure in Figure 7 show the dominant exponentials which correspond to the D-solitons of $\left[i_{1}, j_{1}: i_{4}, j_{4}\right]=[1,8: 4,5]$ and $\left[i_{2}, j_{2}: i_{3}, j_{3}\right]=[2,7: 3,6]$.

(ii) For three D-solitons, we take $\mathbf{p}=(-5,-3.5,-2,-1,0,3,4,7)$. The three open circles in the right figure in Figure 7 correspond to the two D-solitons of $\left[i_{1}, j_{1}: i_{2}, j_{2}\right]=[1,8: 2,7]$, $\left[i_{2}, j_{2}: i_{3}, j_{3}\right]=[2,7: 3,6]$ and $\left[i_{2}, j_{2}: i_{4}, j_{4}\right]=[2,7: 4,5]$.

In Figure 8, we show the interaction patterns for those cases shown in Figure 7 (they are topologically the same as in Figure 5).

In Section 5, we will extend Propositions 4.2 and 4.3 to the general cases as Propositions 5.1 and 5.2 .

Recall that a D-soliton can be identified as an element of the Weyl group of D-type, $W^{D}$. In the case of two D-solitons, we have, for example, $\pi_{1,3}:\left(i_{1}, j_{1}\right) \leftrightarrow\left(i_{3}, j_{3}\right)$ and $\pi_{2,4}:\left(i_{2}, j_{2}\right) \leftrightarrow$ $\left(i_{4}, j_{4}\right)$. The group generated by those elements is an abelian subgroup of $W^{D}$, and the orbit of this subgroup of $\left(i_{1}, j_{1}, i_{2}, j_{2}\right)$ represents the asymptotic regions divided by the solitons, i.e. $\left(i_{1}, j_{1}, i_{2}, j_{2}\right),\left(i_{1}, j_{1}, i_{4}, j_{4}\right),\left(i_{2}, j_{2}, i_{3}, j_{3}\right)$ and $\left(i_{3}, j_{3}, i_{4}, j_{4}\right)$, which correspond to the dominant exponentials in the $\tau$-function, that is, $\hat{E}_{1} \hat{E}_{2}, \hat{E}_{1} \hat{E}_{4}, \hat{E}_{2} \hat{E}_{3}$ and $\hat{E}_{3} \hat{E}_{4}$, respectively. In the $\tau$-function, we have two more exponential terms labeled by $\left(i_{1}, j_{1}, i_{3}, j_{3}\right)$ and $\left(i_{2}, j_{2}, i_{4}, j_{4}\right)$, i.e. $\hat{E}_{1} \hat{E}_{3}$ and $\hat{E}_{2} \hat{E}_{4}$. Those terms become dominant at the points of the resonant quadrangle as in the case of two Asolitons of T-type. Namely we have a resonant two D-soliton. The resonant condition is given by, for example,

$$
\left\{\begin{array}{l}
\mathbf{k}\left[i_{1}, j_{1}: i_{3}, j_{3}\right]+\mathbf{k}\left[i_{3}, j_{3}: i_{4}, j_{4}\right]=\mathbf{k}\left[i_{1}, j_{1}: i_{4}, j_{4}\right] \\
\omega\left[i_{1}, j_{1}: i_{3}, j_{3}\right]+\omega\left[i_{3}, j_{3}: i_{4}, j_{4}\right]=\omega\left[i_{1}, j_{1}: i_{4}, j_{4}\right]
\end{array}\right.
$$

In the case of three D-soliton, for example $\left[i_{1}, j_{1}: i_{2}, j_{2}\right],\left[i_{2}, j_{2}: i_{3}, j_{3}\right]$ and $\left[i_{2}, j_{2}: i_{4}, j_{4}\right]$, we have $\pi_{1,2}, \pi_{2,3}$ and $\pi_{2,4}$ as the generating elements of the subgroup. Then the subgroup contains $\pi_{1,3}, \pi_{1,4}$ and $\pi_{3,4}$, and those are the D-solitons generated by the resonances of pairs, i.e. $\pi_{k, l}$ represents D-soliton of $\left[i_{k}, j_{k}: i_{l}, j_{l}\right]$. Each resonant relation can be represented by the product $\pi_{i, 2} \cdot \pi_{2, j}=\pi_{i, j}$.

For any two D-soliton solution in Proposition 4.1, we have:

Proposition 4.4. Two D-solitons constructed above are all in resonance, in the sense that there is a resonant quadrangle at the intersection point. Namely all the cases are of T-type with one hole. 

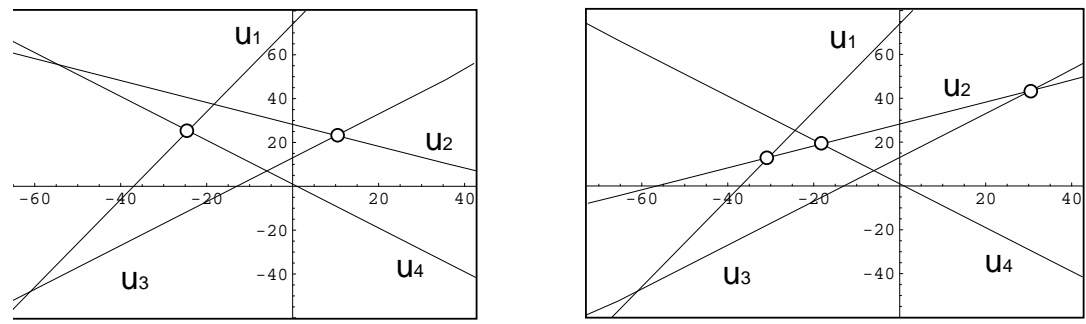

Figure 7. The graphs of $u_{k}(c)=\left(p_{k}+p_{9-k}\right) c+p_{k}^{2}+p_{9-k}^{2}$ for $k=1, \ldots, 4$. The indices are marked by $\left(i_{k}, j_{k}\right)=(k, 9-k)$ for $k=1, \ldots, 4$. The left figure shows two Dsoliton solutions of $[1,8: 4,5]$ and $[2,7: 3,6]$ with $\mathbf{p}=(-5,-4,-2,-1,0,3,3.5,7)$, and the right one shows three D-solitons of $[1,8: 2,7],[2,7: 3,6]$ and $[2,7: 4,5]$ with $\mathbf{p}=(-5,-3.5,-2,-1,0,3,4,7)$. Note that only $p_{2}$ is changed.
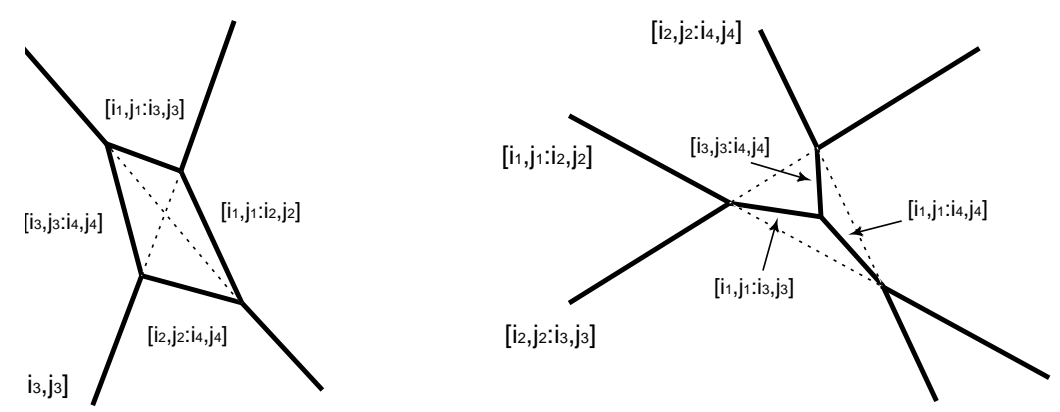

FiguRE 8. Interaction patterns for two and three D-solitons in Figure 7. The function $w=\left(\ln \tau_{2}\right)_{x}$ takes the values, $w \rightarrow\left(i_{1}, j_{1}, i_{2}, j_{2}\right)$ as $x \rightarrow-\infty$, and $w \rightarrow$ $\left(i_{3}, j_{3}, i_{4}, j_{4}\right)$ as $x \rightarrow \infty$. All six D-solitons in each figure can be identified as the six intersection points of the graphs $u_{k}:=u_{i_{k}, j_{k}}$ in Figure 7 .

Proof. First note that there are six exponential terms in the $\tau$-function. Each exponential term becomes dominant in some region in the $x-y$ plane. For example, consider two D-solitons with $[1,2: 5,6]$ and $[3,4: 7,8]$. Then the function $w=\left(\ln \tau_{2}\right)_{x}$ takes $w \rightarrow(1,2,3,4)$ for $x \rightarrow-\infty$, and $w \rightarrow(5,6,7,8)$ for $x \rightarrow \infty$. With those $\mathrm{D}$-solitons, we have the regions marked by $(1,2,7,8)$ and $(3,4,5,6)$. In addition to those exponentials, we also have $(1,2,5,6)$ and $(3,4,7,8)$. As in the case of two solitons of the KP equation (see [2]), a resonant interaction of Y-shape can occur. For example, $[1,2: 5,6],[1,2: 7,8]$ and $[5,6: 7,8]$ solitons are in resonant, and the three regions divided by those solitons are marked by $(1,2,3,4),(3,4,5,6)$ and $(3,4,7,8)$. Then the six exponentials in the $\tau$-function form two D-solitons in resonance (see the left figure in Figure 8).

Remark 4.3. In Figure 8 of [5], Isojima et al presented an example of (two) D-solitons which seems to have only one intermediate soliton (no resonant quadrangle). However, it turns out that this is not two D-solitons: First we note that this is the case $(\mathrm{k})$ in Proposition 4.1, i.e. $\mathbf{i}=(1,2,5,7)$ and $\mathbf{j}=(4,3,6,8)$. This can be found by ordering the parameters $\left(p_{1}, \ldots, p_{4}, q_{1}, \ldots, q_{4}\right)$ in Figure 8 of [5], i.e. relabel $\left(p_{1}, \ldots, q_{4}\right)$ in terms of our ordering $\left(p_{1}, \ldots, p_{8}\right)$ with $p_{1} \rightarrow p_{8}, p_{2} \rightarrow p_{7}, p_{3} \rightarrow p_{4}, p_{4} \rightarrow$ $p_{1}, q_{1} \rightarrow p_{6}, q_{2} \rightarrow p_{5}, q_{3} \rightarrow p_{3}$ and $q_{4} \rightarrow p_{2}$. The parameters chosen in the paper is then given by $\mathbf{p}=\left(-\frac{3}{2},-\frac{4}{3},-\frac{2}{3},-\frac{1}{2}, \frac{1}{2}, \frac{2}{3}, \frac{3}{2}, 3\right)$. With those parameters, we note that $p_{1}+p_{4}=:(1,4)=(2,3)=$ : $p_{2}+p_{3}$, a degeneracy in the parameters. Two D-solitons are given by $[1,4: 5,6]$ and $[2,3: 7,8]$, and the asymptotic values of $w$ are $(1,2,3,4)$ and $(5,6,7,8)$ for $x \rightarrow \mp \infty$. Then the resonant quadrangle consists of the D-solitons of $[1,4: 2,3],[1,4: 7,8],[2,3: 5,6]$ and $[5,6: 7,8]$. However, because of the degeneracy $(1,4)=(2,3)$, the $[1,4: 2,3]$-soliton cannot exist, i.e. the function $w$ cannot change the value across this soliton. Also notice that the $[2,3: 5,6]$ - and $[1,4: 5,6]$-solitons are almost parallel, and so are the $[1,4: 7,8]$ - and $[2,3: 7,8]$-solitons: For example, the peak of the soliton $[2,3: 5,6]$ is 
given by $\theta_{2,3}=\theta_{5,6}$, i.e. for a fixed $t$ and with the values of $\mathbf{p}$, we have the line for $[2,3: 5,6]$-soliton,

$$
\left(p_{5}+p_{6}-p_{2}-p_{3}\right) x+\left(p_{5}^{2}+p_{6}^{2}-p_{2}^{2}-p_{3}^{2}\right) y=\frac{57}{18}\left(x-\frac{55}{114} y\right)=\text { const. }
$$

which is almost parallel to the line $\theta_{1,4}=\theta_{5,6}$ for the $[1,4: 5,6]$-soliton,

$$
\left(p_{5}+p_{6}-p_{1}-p_{4}\right) x+\left(p_{5}^{2}+p_{6}^{2}-p_{1}^{2}-p_{4}^{2}\right) y=\frac{57}{18}\left(x-\frac{65}{114} y\right)=\text { const. }
$$

Thus the soliton solution in Figure 8 of [5] consists of two D-solitons of $[1,4: 5,6]$ and $[2,3: 7,8]$ for $y \rightarrow \infty$ and two other $\mathrm{D}$-solitons of $[2,3: 5,6]$ and $[1,4: 7,8]$ for $y \rightarrow-\infty$, and the intermediate soliton is the $[5,6: 7,8]$-soliton. Other intermediate soliton of $[1,4: 2,3]$ may be considered to be located at $y=-\infty$.

For any three D-soliton solutions in Proposition 4.1, we have:

Proposition 4.5. Any pair of three D-solitons constructed above is in resonance to form an $Y$-shape vertex. This three $D$-soliton solution has no resonant hole and four resonant $Y$-shape vertices.

Proof. Since there are six exponential terms which mark the six separated regions in the $x-y$ plane bounded by those three solitons, this 3 -soliton solution cannot have a resonant hole. Three solitons are labeled by, for example, $\left[i_{1}, j_{1}: i_{2}, j_{2}\right],\left[i_{2}, j_{2}: i_{3}, j_{3}\right]$ and $\left[i_{2}, j_{2}: i_{4}, j_{4}\right]$, and any pair of those solitons is in resonance, i.e. $\left[i_{1}, j_{1}: i_{2}, j_{2}\right]$ and $\left[i_{2}, j_{2}: i_{3}, j_{3}\right]$ have a resonant interaction to generate $\left[i_{1}, j_{1} ; i_{3}, j_{3}\right]$-soliton (see the right figure in Fig.8). It is easy to see that this resonance appear for any three solitons obtained in Proposition 4.1.

4.2. Three soliton solutions consisting of two A-solitons and one D-soliton. Since one D-soliton is a degenerate two A-solitons, one can construct 3-soliton solution consisting of two Asolitons and one D-soliton from two D-solitons. One should note that resolution of one D-soliton into two A-solitons can work only for the case of two D-solitons. In the case of three D-solitons, the resolution of one D-soliton affects to other D-solitons, and it cannot produce two A-solitons. This can be checked by the asymptotic analysis using the graphs of $u_{i, j}$.

Example 4.4. Consider two D-solitons corresponding to the $B$-matrix of the case (n) in Proposition 4.1, i.e. $\mathbf{i}=(1,3,5,7)$ and $\mathbf{j}=(2,4,6,8)$. Two D-solitons are $[1,2: 5,6]$ and $[3,4: 7,8]$. Then, for example, two A-soliton of type $[1: 5]$ and $[2: 6]$ are obtained by adding two extra nonzero elements at $(1,6)$ and $(2,5)$. This fact can be verified easily from the diagram of T-type (up-side-down staircase with sides labeled by 1,2,5 and 6; see Figure 3). The 3-solitons obtained here has only one hole (see Figure 9).

One can also get two A-solitons of $[1: 6]$ and $[2: 5]$ from $[1,2: 5,6]$-soliton by adding two nonzero elements at $(1,5)$ and $(2,6)$. Notice that those two A-solitons form the diagram of P-type (cannon shape with sides by $1,2,5$ and 6 ; see Figure 3 ).

In the same way, one can resolve $[3,4: 7,8]$-solitons into either the pair of $[3: 7]$ and $[4: 8]$ or the pair of $[3: 8]$ and $[4: 7]$, by adding extra nonzero entries to the $B$-matrix of the case (n).

4.3. Four A-soliton solutions from D-solitons. Those solitons can be constructed in the similar way as the case of three solitons (two A-solitons and one D-soliton) from two D-solitons. However, the compatibility among those A-solitons separated from D-solitons is somewhat complicated. For example, one can expect that two D-solitons, $[1,2: 5,6]$ and $[3,4: 7,8]$, generate two A-solitons $[1: 5],[2: 6]$ from $[1,2: 5,6]$ and other two A-solitons $[3: 7]$ and $[4: 8]$ from $[3,4: 7,8]$. The $B$-matrices for 3 -solitons $[1: 5],[2: 6],[3,4: 7,8]$ and $[1,2: 5,6],[3: 7],[4: 8]$ may be respectively 

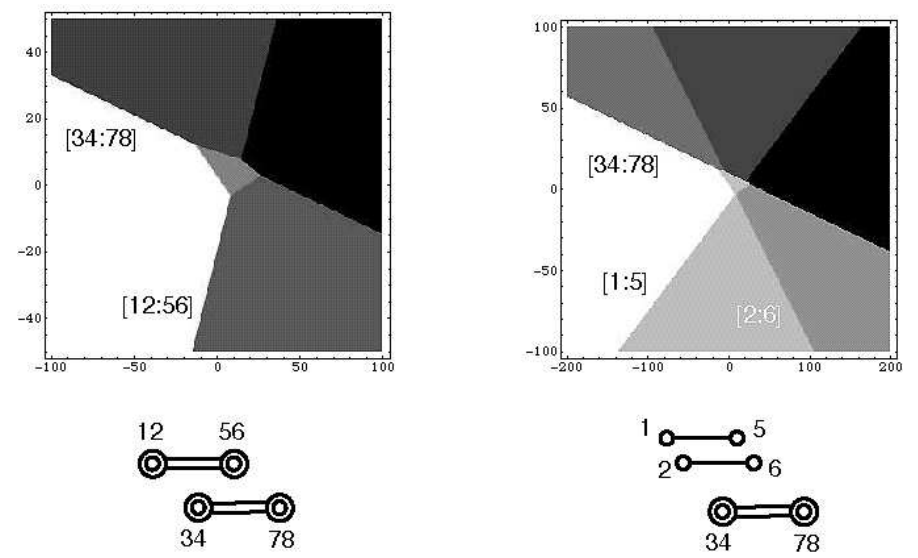

Figure 9. Bifurcation of two D-solitons into three solitons with two A-solitons and one D-soliton. The $[1,2: 5,6]$-D-soliton resolves into two A-solitons of $[1: 5]$ and $[2: 6]$. D-solitons are expressed as the double lines joined with the double circles.

given by

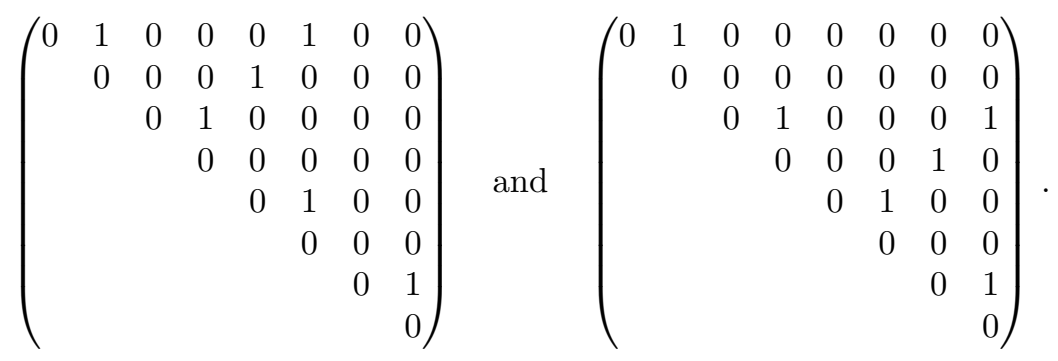

Then adding $b_{3,8}, b_{4,7}$ to the $B$-matrix in the left (or $b_{1,6}, b_{2,5}$ to the right one), the resulting $B$ matrix is expected to give four A-solitons $[1: 5],[2: 6],[3: 7]$ and $[4: 8]$. However this matrix gives a singular solution, and a correct $B$-matrix for this 4 -soliton solution may be given by

$$
\left(\begin{array}{llllllll}
0 & 1 & 0 & 0 & 0 & 0 & 0 & 1 \\
& 0 & 1 & 0 & 0 & 0 & 0 & 0 \\
& & 0 & 1 & 0 & 0 & 0 & 0 \\
& & & 0 & 1 & 0 & 0 & 0 \\
& & & & 0 & 1 & 0 & 0 \\
& & & & & 0 & 1 & 0 \\
& & & & & & 0 & 1 \\
& & & & & & & 0
\end{array}\right)
$$

Thus in this case we do not have a compatibility between the two sets of 3 solitons obtained from the D-solitons (in the sense that the $\tau$-function cannot stay to be sign-definite). A general rule to construct a $B$-matrix for four A-solitons from two D-solitons seems to be complicated. Here we give a direct way to construct a $B$-matrix for a given four A-solitons, based on the $B$-matrices obtained in Proposition 4.1:

1) Give four A-solitons labeled by a pair of four numbers, i.e.

$$
\mathbf{n}^{-}=\left(n_{1}^{-}, \ldots, n_{4}^{-}\right), \quad \mathbf{n}^{+}=\left(n_{1}^{+}, \ldots, n_{4}^{+}\right)
$$

where $1=n_{1}^{-}<\cdots<n_{4}^{-}$and $n_{i}^{-}<n_{i}^{+}$. Then each soliton is labeled by $\left[n_{k}^{-}: n_{k}^{+}\right]$for $k=1, \ldots, 4$ (the same as in the $\mathrm{KP}$ solitons [8]). This implies that the asymptotic values of $w=(\ln \tau)_{x}$ are given by $\sum_{k=1}^{4} p_{n_{k}^{ \pm}}$for $x \rightarrow \pm \infty$.

2) Take a pair of two solitons, say $\left[n_{i}^{-}: n_{i}^{+}\right]$and $\left[n_{j}^{-}: n_{j}^{+}\right]$, and draw the corresponding diagrams shown in Figure 3, and do the same for the other pair. Here the choice of the pair 
should be consistent with the asymptotic values. Then adjust the signs for each horizontal edges of the diagrams, so that all the pfaffians have the same sign. If all are satisfied, then the diagrams give the $B$-matrix.

Example: Consider four solitons with $[1: 2],[7: 8]$ and any other two solitons with the indices from $\{3,4,5,6\}$. Then one can construct the $B$-matrix as follows:

The diagram corresponding to $[1: 2],[7: 8]$ is a backward C-shape (P-type shown in Figure 3 ), having $1,2,7$ and 8 on the vertical edge. This implies we have nonzero elements for $b_{1,7}, b_{1,8}, b_{2,7}, b_{2,8}$. Now if the other pair is $[3: 6],[4: 5]$, then the diagram associated to this is a cannon-shape (Otype) having $3,4,5,6$ on the vertical edges. This gives the nonzero entries $b_{3,4}, b_{3,5}, b_{4,6}, b_{5,6}$. In this example, all those entries in the upper triangular part can take +1 for a sign-definite $\tau$-function. Namely we have

$$
B=\left(\begin{array}{llllllll}
0 & 0 & 0 & 0 & 0 & 0 & 1 & 1 \\
& 0 & 0 & 0 & 0 & 0 & 1 & 1 \\
& & 0 & 1 & 1 & 0 & 0 & 0 \\
& & & 0 & 0 & 1 & 0 & 0 \\
& & & & 0 & 1 & 0 & 0 \\
& & & & & 0 & 0 & 0 \\
& & & & & & 0 & 0 \\
& & & & & & & 0
\end{array}\right)
$$

In the case of two solitons of T-type, [3:5], [4:6], one can use the $B$-matrix having the same entries except $b_{3,5}=2$, and in addition $b_{3,6}=1, b_{4,5}=1$.

Notice that the nonzero entries has two groups corresponding to two D-solitons $[1,7: 2,8]$ and $[3,4: 5,6]$, and those groups have no partial overlaps. This structure is true for any cases satisfying the nonsigular condition, i.e. all the pfaffians have the same sign. (Recall that the $B$-matrix with $b_{1,8}=b_{2,7}=b_{3,4}=b_{5,6}=1$, i.e. $\mathbf{i}=(1,2,3,5), \mathbf{j}=(8,7,4,6)$, also gives three D-solitons of $[1,8: 2,7],[2,7: 3,4]$ and $[2,7: 5,6]$.

For the same 4 -solitons as the previous example, i.e. $[1: 2],[3: 6],[4: 5]$ and $[7: 8]$, one can also have the following $B$-matrix which gives the same $\tau_{2}$ function,

$$
B=\left(\begin{array}{cccccccc}
0 & 0 & 0 & 1 & 1 & 0 & 0 & 0 \\
& 0 & 0 & 1 & 1 & 0 & 0 & 0 \\
& & 0 & 0 & 0 & 0 & -1 & -1 \\
& & & 0 & 0 & 0 & 0 & 0 \\
& & & & 0 & 0 & 0 & 0 \\
& & & & & 0 & 1 & 1 \\
& & & & & & 0 & 0 \\
& & & & & & & 0
\end{array}\right) .
$$

This matrix can be constructed from the $B$-matrix for $\mathbf{i}=(1,2,3,6)$ and $\mathbf{j}=(5,4,7,8)$, the case (c) in Proposition 4.1, (i.e. this gives two D-solitons with $[1,5: 2,4]$ and $[3,7: 6,8]$ ). In Figure 10, we show the example of four A-solitons based on two $B$-matrices: The case a) corresponds to the $B$-matrix for $\mathbf{i}=(1,2,3,6), \mathbf{j}=(5,4,7,8)$, and the case b) for $\mathbf{i}=(1,2,3,5), \mathbf{j}=(8,7,4,6)$. We take $\mathbf{p}=(-4,-3,-2,-1,1,3,4,6)$ for both cases.

As an example of four A-solitons, we consider the case with $[1: 7],[2: 8],[3: 5]$ and $[4: 6]$, i.e. there are two partial overlaps (two solitons of T-type). We first note that this set of 4 -solitons can be split into two groups of 2-solitons having no partial overlaps, $\{[1: 7],[2: 8]\}$ and $\{[3: 5],[4: 6]\}$. Since they are both TD-type, we have the TD-type diagram (up-side-down staircase). This implies we have nonzero entries $b_{1,8}, b_{1,2}, b_{2,7}, b_{7,8}$ from the set $\{[1: 7],[2: 8]\}$, and $b_{3,4}, b_{3,6}, b_{4,5}, b_{5,6}$ from the second set. However this $B$-matrix does not give the 4 -solitons. In Figure 11, the left figure shows the solution given by this $B$-matrix, two D-solitons, $[1,2: 5,6]$ and $[3,4: 7,8]$ with some additional resonances at the intersection region of those solitons. This can be explained as follows: This $B$-matrix can be considered as a deformation (bifurcation) from the $B$-matrix of two D-solitons, $[1,2: 5,6]$ and $[3,4: 7,8]$, and the $\tau$-function with this $B$-matrix gives resonant D-solitons, $[1,2$ : 


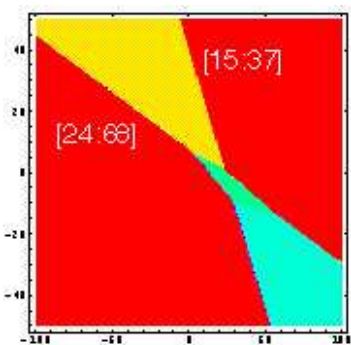

a)

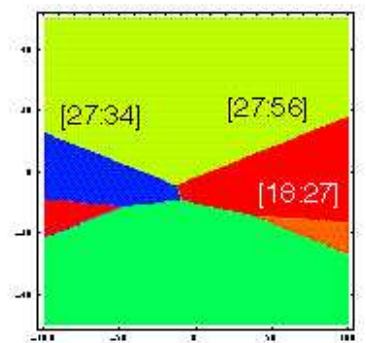

b)

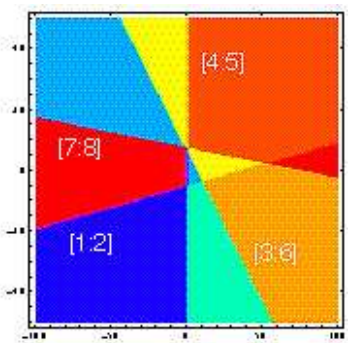

c)

Figure 10. Four A-solitons obtained from the $B$-matrix of two D-solitons or three D-solitons. Two D-solitons in a) is given by the $B$-martrix with $\mathbf{i}=(1,2,3,6)$ and $\mathbf{j}=(5,4,7,8)$. Three D-solitons in $\mathrm{b})$ is given by the $B$-matrix with $\mathbf{i}=(1,2,3,5)$ and $\mathbf{j}=(8,7,4,6)$. Both cases have the same $\mathbf{p}=(-4,-3,-2,-1,1,3,4,6)$. The four A-soliton solution in c) is given by the $B$-matrix (4.1). Note that the $B$-matrix of (4.2) gives the same solution.

$3,4],[1,2: 7,8],[3,4: 5,6]$ and $[5,6: 7,8]$. Then TD-type of 2-solitons [1:7], [2:8] is obtained from the bifurcation of $[1,2: 7,8]$-soliton, which is one of the resonant solitons. Also note that the exponentials associated to $(1,2,7,8)$ and $(3,4,5,6)$ become dominant for two D-solitons for either $y \rightarrow \infty$ or $y \rightarrow-\infty$. However those exponentials should not appear for this set of four A-solitons, that is, those four A-solitons cannot be obtained from this $B$-matrix. This can be fixed by taking following $B$-matrix (i.e. change TD-type to T-type),

$$
B=\left(\begin{array}{llllllll}
0 & 1 & 0 & 0 & 0 & 0 & 2 & 1 \\
& 0 & 0 & 0 & 0 & 0 & 1 & 1 \\
& & 0 & 1 & 2 & 1 & 0 & 0 \\
& & & 0 & 1 & 1 & 0 & 0 \\
& & & & 0 & 1 & 0 & 0 \\
& & & & & 0 & 0 & 0 \\
& & & & & & 0 & 1 \\
& & & & & & & \\
& & & & & & &
\end{array}\right) .
$$

In Figure 11, the right figure shows the 4 -solitons generated by this $B$-matrix.

We also mention that the $\tau_{2}$-function with the generic $8 \times 8 \mathrm{~B}$-matrix having all nonzero entries gives four A-solitons of T-type, that is, they are the same type of the 4-soliton solution of the KP equation. Just notice that the number of exponential terms in $\tau_{2}$ is $\left(\begin{array}{l}8 \\ 4\end{array}\right)=70$, which is the same as the $\tau$-function for the KP equation (see [2]). Then the asymptotic analysis of the $\tau$-functions shows that four A-solitons are given by $[1: 5],[2: 6],[3: 7]$ and $[4: 8]$, which are of T-type. An example of the generic $B$-matrix for nonsingular $\tau$-function is given by the skew-symmetric matrix having 1 's in all the entries in the upper triangular part.

\section{Multi-Soliton SOlutions}

For the general case with arbitrary $M=4 N$, we consider the $B$-matrix with $2 N$ numbers of nozero entries $b_{i_{k}, j_{k}}$ for $k=1, \ldots, N$ and $1=i_{1}<\cdots<i_{N}, i_{k}<j_{k}$ in the upper triangular part. Although there is a scheme to find the $B$-matrix for sign-definite $\tau_{N}$ (i.e. having all the same sign in the pfaffian coefficients), the generalization of Proposition 4.1 seems to be difficult. However, we can show the following Propositions regarding to the number of D-solitons for two special cases:

Proposition 5.1. The $\tau_{N}$-function (1.2) with $4 N \times 4 N$ B-matrix given by $J_{0}$ of (2.1) can have only $N$ D-solitons in the generic situation. 

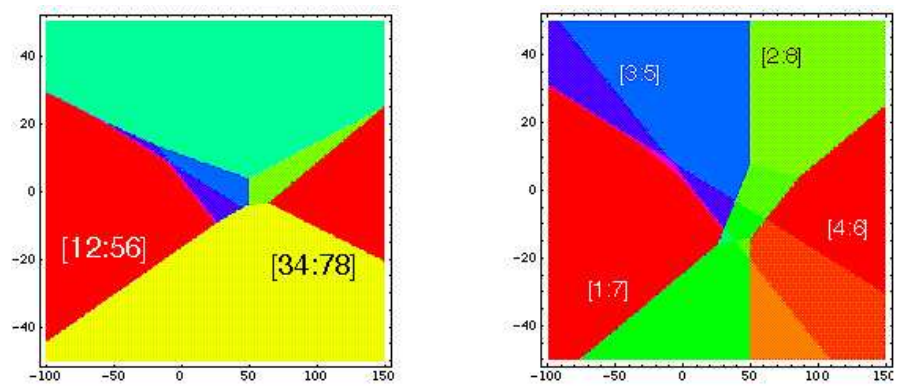

Figure 11. The 4-solutions in the right figure is obtained from the $B$-matrices given by (4.3). The left figure shows two D-solitons obtained by the same matrix except $b_{1,7}=0, b_{2,8}=0, b_{3,5}=0$ and $b_{4,6}=0$ (note the extra resonant structure at the intesection region of those D-solitons.

Proof. Apply Lemma 4.2, and find the dominant exponentials by following the argument of "level of intersection" introduced in [2] (the main idea here is to find the dominant exponents from the graphs of $\left.u_{i, j}(c)\right)$.

The other case corresponds to the $B$-matrix, $B=J_{\pi}$, where $J_{\pi}$ is defined by $J_{\pi}:=\pi J_{0} \pi^{T}$ with $\pi \in S_{4 N}$, the permutation given by $\pi=[2: 4 N] \cdot[4: 4 N-2] \cdots[2 N: 2 N+2]$, i.e.

$$
J_{w}=\left(\begin{array}{cccccccc}
0 & 0 & \cdots & 0 & 0 & \cdots & 0 & 1 \\
& 0 & \cdots & 0 & 0 & \cdots & 1 & 0 \\
& & \ddots & \vdots & \vdots & \vdots & \vdots & \vdots \\
& & & 0 & 1 & \cdots & 0 & 0 \\
& & & & 0 & \cdots & 0 & 0 \\
& & & & & \ddots & \vdots & \vdots \\
& & & & & & 0 & 0 \\
& & & & & & & 0
\end{array}\right)
$$

Then we have:

Proposition 5.2. The number of D-solitons generated by the $\tau_{N}$-function (1.2) with $4 N \times 4 N B$ matrix given by $J_{w}$ of (5.1) can be any number from $N$ to $2 N-1$, depending on the choice of the parameter $\mathbf{p}$.

Here instead of giving a precise proof, we demonstrate a case with $M=16(N=4)$ : In Figure 12 , we show the graphs of $u_{k}(c)=\left(p_{k}+p_{17-k}\right) c+p_{k}^{2}+p_{17-k}^{2}$ for $k=1, \ldots, 8$. With a proper choice of the parameter $\mathbf{p}=\left(p_{1}, \ldots, p_{16}\right)$, one can find four different cases of intersection patterns of those lines. Then D-solitons can be found at the intersection points marked by open circles in Figure 12, that is, the level of intersection of those points should be four (i.e. there are four lines above those points). For example, D-soliton $[4,13: 5,12]$ is obtained by the intersection of $u_{4}$ and $u_{5}$, and two dominant exponents for this soliton are $\hat{E}_{1} \hat{E}_{2} \hat{E}_{3} \hat{E}_{4}$ and $\hat{E}_{1} \hat{E}_{2} \hat{E}_{3} \hat{E}_{5}$ which are obtained from four lines above the intersection point $u_{4}=u_{5}$ (this is the definition of the level of intersection; see [2] for more details). We have the following four cases:

(a) Four D-solitons (the top left figure) of $[1,16: 8,9],[2,15: 7,10],[3,14: 6,11]$ and $[4,13: 5,12]$ with the parameter,

$$
\mathbf{p}=(-16,-14,-12.5,-11,-8.5,-6,-4,-2,2,5,6,9.5,11.2,13.5,14,17) .
$$

(b) Five D-solitons (the top right figure) of $[1,16: 8,9],[2,15: 4,13],[3,14: 6,11],[4,13: 5,12]$ and $[4,13: 7,10]$ with

$$
\mathbf{p}=(-16,-14,-12.5,-11.6,-8.5,-6,-3,-2,2,4,6,9.5,12,13.5,14,17)
$$



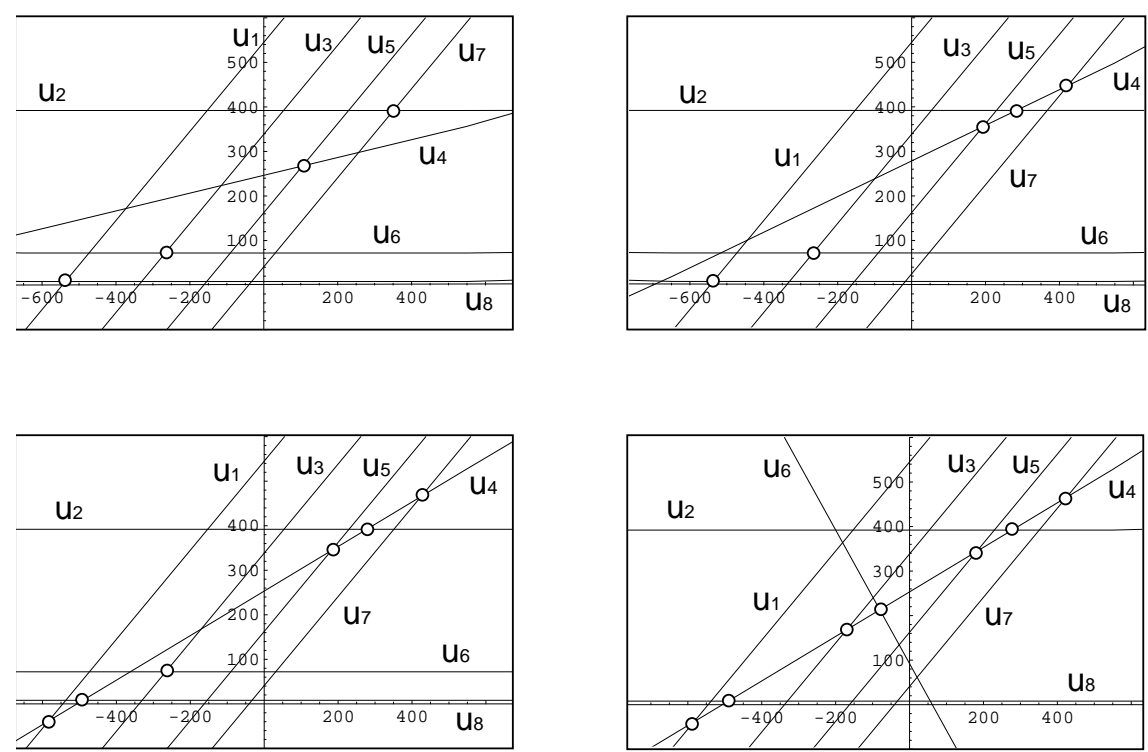

Figure 12. The graphs of $u_{k}(c)=\left(p_{k}+p_{17-k}\right) c+p_{k}^{2}+p_{17-k}^{2}$ for $k=1, \ldots, 16$. Each open circle of the intersections of two functions $u_{k}$ corresponds to a D-soliton given by $\tau_{4}$. For eaxmple, the point for $u_{1}=u_{8}$ corresponds to the D-soliton of $[1,16: 8,9]$. The number of open circles depends on the choice of the parameter $\mathbf{p}=\left(p_{1}, \ldots, p_{16}\right)$. Notice that only $u_{4}$ and later $u_{6}$ are changed to increase the number of solitons (see the text for the explicit values of $\mathbf{p}$ ).

Note here that three $\mathrm{D}$-solitons have the common index $(4,13)$. The $[2,15: 7,10]$-soliton in the case (a) causes the resonant interaction with two D-solitons $[2,15: 4,13]$ and $[4,13$ : $7,10]$.

(c) Six D-solitons (the bottom left figure) of [1, $16: 4: 13],[2,15: 4,13],[3,14: 6,11],[4,13$ : $5,12],[4,13: 7,10]$ and $[4,13: 8,9]$ with

$$
\mathbf{p}=(-16,-14,-12.5,-11,-8.5,-6,-4,-2,2,5,6,9.5,11.5,13.5,14,17) .
$$

(d) Seven D-solitons (the bottom right figure) of $[k, 17-k: 4,13]$ for all $k=1, \ldots, 8$, except $k=4$, and

$$
\mathbf{p}=(-16,-14,-12.5,-11,-8.5,-7.5,-4,-2,2,5,6,9.5,11.5,13.5,14,17) .
$$

One should note that those $\mathbf{p}$ values do not give generic cases, since some of the lines $u_{k}(c)$ are parallel as shown in Figure 12. However, one can make those to be generic by changing slightly the parameters $p_{i}$ 's without breaking the intersection patterns. (We show those non-generic parameters, since those provide a clear evidence of the existence of those solitons.)

We also remark that all the $N$-soliton solutions obtaind by $\tau_{N}$ with the $B$-matrix given in Proposition 5.1 are fully resonant cases like the T-type for the KP equation, that is, the $\tau_{N}$ contains $\left(\begin{array}{c}2 N \\ N\end{array}\right)$ exponential terms having the form $\prod_{k=1}^{N} \hat{E}_{k}=\prod_{k=1}^{N} E_{i_{k}, j_{k}}$ (see [2]).

Finally, we note that in the case of generic $B$-matrix having all nonzero entries, the $\tau_{N}$ function has the maximal number of exponential terms $\left(\begin{array}{l}4 N \\ 2 N\end{array}\right)$ which has the same structure of the $\tau$-function for fully resonant $2 N$-soliton solutions (i.e. T-type) of the KP equation. Those $2 N$ solitons are all A-type, and the interaction patterns are topologically the same for both KP and DKP equations. In those cases, the functions $v^{ \pm}$appear only locally at the interaction regions among $2 N$-solitons.

\section{REFERENCES}

[1] M. Adler, E. Horozov and P. van Moerbeke, The Pfaff lattice and skew-orthogonal polynomials, Intern. Math. Res. Notices, 11 (1999) 569-588. 
[2] G. Biondini and Y. Kodama, On a family of solutions of the Kadomtsev-Petviashvili equation which also satisfy the Toda lattice hierarchy, J. Phys. A: Math. Gen. 36 (2003) 10519-36.

3] R. Hirota and Y. Ohta, Hierarchies of coupled soliton equations I, J. Phys. Soc. Japan, 60 (1991) 798-809.

[4] M. Ishikawa and M. Wakayama, Minor summation formula of pfaffians, Linear and Multilinear Algebra, 39 (1995) 285-305.

[5] S. Isojima, R. Willox and J. Satsuma, On various solutions of the coupled KP equation, J. Phys. A: Math. Gen., 35 (2002) 6893-6909.

[6] M. Jimbo and T. Miwa, Solitons and infinite dimensional Lie algebras, Publ. Res. Inst. Math. Sci. Kyoto University, 19 (1983) 943-1001.

[7] S. Kakei, Orthogonal and symplectic matrix integrals and coupled KP hierarchy, J. Phys. Soc. of Japan, 68 (1999) 2875-2877.

[8] Y. Kodama, Young diagrams and $N$-soliton solutions of the KP equation, J. Phys. A: Math. Gen., 37 (2004) $11169-11190$

[9] V. G. Kac and J. W. van de Leur, The geometry of spinors and the multicomponent BKP and DKP hierarchies. The Bispectral Problem. CRM Proc. Lecture Notes 14, AMS, Providence, (1998) 159-202.

Department of Mathematics, Ohio State University, Columbus, OH 43210

E-mail address: kodama@math.ohio-state.edu

Faculty of Mathematics, Kyushu University, Fukuoka, 812-8581, Japan

E-mail address: maruno@math.kyushu-u.ac.jp 


\section{List of MHF Preprint Series, Kyushu University 21st Century COE Program Development of Dynamic Mathematics with High Functionality}

\section{MHF2003-1 Mitsuhiro T. NAKAO, Kouji HASHIMOTO \& Yoshitaka WATANABE}

A numerical method to verify the invertibility of linear elliptic operators with applications to nonlinear problems

MHF2003-2 Masahisa TABATA \& Daisuke TAGAMI

Error estimates of finite element methods for nonstationary thermal convection problems with temperature-dependent coefficients

MHF2003-3 Tomohiro ANDO, Sadanori KONISHI \& Seiya IMOTO

Adaptive learning machines for nonlinear classification and Bayesian information criteria

MHF2003-4 Kazuhiro YOKOYAMA

On systems of algebraic equations with parametric exponents

MHF2003-5 Masao ISHIKAWA \& Masato WAKAYAMA

Applications of Minor Summation Formulas III, Plücker relations, Lattice paths and Pfaffian identities

MHF2003-6 Atsushi SUZUKI \& Masahisa TABATA

Finite element matrices in congruent subdomains and their effective use for large-scale computations

MHF2003-7 Setsuo TANIGUCHI

Stochastic oscillatory integrals - asymptotic and exact expressions for quadratic phase functions -

MHF2003-8 Shoki MIYAMOTO \& Atsushi YOSHIKAWA

Computable sequences in the Sobolev spaces

MHF2003-9 Toru FUJII \& Takashi YANAGAWA

Wavelet based estimate for non-linear and non-stationary auto-regressive model

MHF2003-10 Atsushi YOSHIKAWA

Maple and wave-front tracking - an experiment

MHF2003-11 Masanobu KANEKO

On the local factor of the zeta function of quadratic orders

MHF2003-12 Hidefumi KAWASAKI

Conjugate-set game for a nonlinear programming problem 
MHF2004-1 Koji YONEMOTO \& Takashi YANAGAWA

Estimating the Lyapunov exponent from chaotic time series with dynamic noise

MHF2004-2 Rui YAMAGUCHI, Eiko TSUCHIYA \& Tomoyuki HIGUCHI

State space modeling approach to decompose daily sales of a restaurant into time-dependent multi-factors

MHF2004-3 Kenji KAJIWARA, Tetsu MASUDA, Masatoshi NOUMI, Yasuhiro OHTA \& Yasuhiko YAMADA

Cubic pencils and Painlevé Hamiltonians

MHF2004-4 Atsushi KAWAGUCHI, Koji YONEMOTO \& Takashi YANAGAWA

Estimating the correlation dimension from a chaotic system with dynamic noise

MHF2004-5 Atsushi KAWAGUCHI, Kentarou KITAMURA, Koji YONEMOTO, Takashi YANAGAWA \& Kiyofumi YUMOTO

Detection of auroral breakups using the correlation dimension

MHF2004-6 Ryo IKOTA, Masayasu MIMURA \& Tatsuyuki NAKAKI

A methodology for numerical simulations to a singular limit

MHF2004-7 Ryo IKOTA \& Eiji YANAGIDA

Stability of stationary interfaces of binary-tree type

MHF2004-8 Yuko ARAKI, Sadanori KONISHI \& Seiya IMOTO

Functional discriminant analysis for gene expression data via radial basis expansion

MHF2004-9 Kenji KAJIWARA, Tetsu MASUDA, Masatoshi NOUMI, Yasuhiro OHTA \& Yasuhiko YAMADA

Hypergeometric solutions to the $q$ - Painlevé equations

MHF2004-10 Raimundas VIDŪNAS

Expressions for values of the gamma function

MHF2004-11 Raimundas VIDŪNAS

Transformations of Gauss hypergeometric functions

MHF2004-12 Koji NAKAGAWA \& Masakazu SUZUKI

Mathematical knowledge browser

MHF2004-13 Ken-ichi MARUNO, Wen-Xiu MA \& Masayuki OIKAWA

Generalized Casorati determinant and Positon-Negaton-Type solutions of the Toda lattice equation

MHF2004-14 Nalini JOSHI, Kenji KAJIWARA \& Marta MAZZOCCO

Generating function associated with the determinant formula for the solutions of the Painlevé II equation 
MHF2004-15 Kouji HASHIMOTO, Ryohei ABE, Mitsuhiro T. NAKAO \& Yoshitaka WATANABE

Numerical verification methods of solutions for nonlinear singularly perturbed problem

MHF2004-16 Ken-ichi MARUNO \& Gino BIONDINI

Resonance and web structure in discrete soliton systems: the two-dimensional Toda lattice and its fully discrete and ultra-discrete versions

MHF2004-17 Ryuei NISHII \& Shinto EGUCHI

Supervised image classification in Markov random field models with Jeffreys divergence

MHF2004-18 Kouji HASHIMOTO, Kenta KOBAYASHI \& Mitsuhiro T. NAKAO

Numerical verification methods of solutions for the free boundary problem

MHF2004-19 Hiroki MASUDA

Ergodicity and exponential $\beta$-mixing bounds for a strong solution of Lévydriven stochastic differential equations

MHF2004-20 Setsuo TANIGUCHI

The Brownian sheet and the reflectionless potentials

MHF2004-21 Ryuei NISHII \& Shinto EGUCHI

Supervised image classification based on AdaBoost with contextual weak classifiers

MHF2004-22 Hideki KOSAKI

On intersections of domains of unbounded positive operators

MHF2004-23 Masahisa TABATA \& Shoichi FUJIMA

Robustness of a characteristic finite element scheme of second order in time increment

MHF2004-24 Ken-ichi MARUNO, Adrian ANKIEWICZ \& Nail AKHMEDIEV

Dissipative solitons of the discrete complex cubic-quintic Ginzburg-Landau equation

MHF2004-25 Raimundas VIDŪNAS

Degenerate Gauss hypergeometric functions

MHF2004-26 Ryo IKOTA

The boundedness of propagation speeds of disturbances for reaction-diffusion systems

MHF2004-27 Ryusuke KON

Convex dominates concave: an exclusion principle in discrete-time Kolmogorov systems 
MHF2004-28 Ryusuke KON

Multiple attractors in host-parasitoid interactions: coexistence and extinction

MHF2004-29 Kentaro IHARA, Masanobu KANEKO \& Don ZAGIER

Derivation and double shuffle relations for multiple zeta values

MHF2004-30 Shuichi INOKUCHI \& Yoshihiro MIZOGUCHI

Generalized partitioned quantum cellular automata and quantization of classical CA

MHF2005-1 Hideki KOSAKI

Matrix trace inequalities related to uncertainty principle

MHF2005-2 Masahisa TABATA

Discrepancy between theory and real computation on the stability of some finite element schemes

MHF2005-3 Yuko ARAKI \& Sadanori KONISHI

Functional regression modeling via regularized basis expansions and model selection

MHF2005-4 Yuko ARAKI \& Sadanori KONISHI

Functional discriminant analysis via regularized basis expansions

MHF2005-5 Kenji KAJIWARA, Tetsu MASUDA, Masatoshi NOUMI, Yasuhiro OHTA \& Yasuhiko YAMADA

Point configurations, Cremona transformations and the elliptic difference Painlevé equations

MHF2005-6 Kenji KAJIWARA, Tetsu MASUDA, Masatoshi NOUMI, Yasuhiro OHTA \& Yasuhiko YAMADA

Construction of hypergeometric solutions to the $q$ - Painlevé equations

MHF2005-7 Hiroki MASUDA

Simple estimators for non-linear Markovian trend from sampled data:

I. ergodic cases

MHF2005-8 Hiroki MASUDA \& Nakahiro YOSHIDA

Edgeworth expansion for a class of Ornstein-Uhlenbeck-based models

MHF2005-9 Masayuki UCHIDA

Approximate martingale estimating functions under small perturbations of dynamical systems

MHF2005-10 Ryo MATSUZAKI \& Masayuki UCHIDA

One-step estimators for diffusion processes with small dispersion parameters from discrete observations

MHF2005-11 Junichi MATSUKUBO, Ryo MATSUZAKI \& Masayuki UCHIDA

Estimation for a discretely observed small diffusion process with a linear drift 
MHF2005-12 Masayuki UCHIDA \& Nakahiro YOSHIDA

AIC for ergodic diffusion processes from discrete observations

MHF2005-13 Hiromichi GOTO \& Kenji KAJIWARA

Generating function related to the Okamoto polynomials for the Painlevé IV equation

MHF2005-14 Masato KIMURA \& Shin-ichi NAGATA

Precise asymptotic behaviour of the first eigenvalue of Sturm-Liouville problems with large drift

MHF2005-15 Daisuke TAGAMI \& Masahisa TABATA

Numerical computations of a melting glass convection in the furnace

MHF2005-16 Raimundas VIDŪNAS

Normalized Leonard pairs and Askey-Wilson relations

MHF2005-17 Raimundas VIDŪNAS

Askey-Wilson relations and Leonard pairs

MHF2005-18 Kenji KAJIWARA \& Atsushi MUKAIHIRA

Soliton solutions for the non-autonomous discrete-time Toda lattice equation

MHF2005-19 Yuu HARIYA

Construction of Gibbs measures for 1-dimensional continuum fields

MHF2005-20 Yuu HARIYA

Integration by parts formulae for the Wiener measure restricted to subsets in $\mathbb{R}^{d}$

MHF2005-21 Yuu HARIYA

A time-change approach to Kotani's extension of Yor's formula

MHF2005-22 Tadahisa FUNAKI, Yuu HARIYA \& Mark YOR

Wiener integrals for centered powers of Bessel processes, I

MHF2005-23 Masahisa TABATA \& Satoshi KAIZU

Finite element schemes for two-fluids flow problems

MHF2005-24 Ken-ichi MARUNO \& Yasuhiro OHTA

Determinant form of dark soliton solutions of the discrete nonlinear Schrödinger equation

MHF2005-25 Alexander V. KITAEV \& Raimundas VIDŪNAS

Quadratic transformations of the sixth Painlevé equation

MHF2005-26 Toru FUJII \& Sadanori KONISHI

Nonlinear regression modeling via regularized wavelets and smoothing parameter selection 
MHF2005-27 Shuichi INOKUCHI, Kazumasa HONDA, Hyen Yeal LEE, Tatsuro SATO, Yoshihiro MIZOGUCHI \& Yasuo KAWAHARA

On reversible cellular automata with finite cell array

MHF2005-28 Toru KOMATSU

Cyclic cubic field with explicit Artin symbols

MHF2005-29 Mitsuhiro T. NAKAO, Kouji HASHIMOTO \& Kaori NAGATOU

A computational approach to constructive a priori and a posteriori error

estimates for finite element approximations of bi-harmonic problems

MHF2005-30 Kaori NAGATOU, Kouji HASHIMOTO \& Mitsuhiro T. NAKAO

Numerical verification of stationary solutions for Navier-Stokes problems

MHF2005-31 Hidefumi KAWASAKI

A duality theorem for a three-phase partition problem

MHF2005-32 Hidefumi KAWASAKI

A duality theorem based on triangles separating three convex sets

MHF2005-33 Takeaki FUCHIKAMI \& Hidefumi KAWASAKI

An explicit formula of the Shapley value for a cooperative game induced from the conjugate point

MHF2005-34 Hideki MURAKAWA

A regularization of a reaction-diffusion system approximation to the two-phase

Stefan problem

MHF2006-1 Masahisa TABATA

Numerical simulation of Rayleigh-Taylor problems by an energy-stable finite element scheme

MHF2006-2 Ken-ichi MARUNO \& G R W QUISPEL

Construction of integrals of higher-order mappings

MHF2006-3 Setsuo TANIGUCHI

On the Jacobi field approach to stochastic oscillatory integrals with quadratic phase function

MHF2006-4 Kouji HASHIMOTO, Kaori NAGATOU \& Mitsuhiro T. NAKAO

A computational approach to constructive a priori error estimate for finite element approximations of bi-harmonic problems in nonconvex polygonal domains

MHF2006-5 Hidefumi KAWASAKI

A duality theory based on triangular cylinders separating three convex sets in $R^{n}$

MHF2006-6 Raimundas VIDŪNAS

Uniform convergence of hypergeometric series 
MHF2006-7 Yuji KODAMA \& Ken-ichi MARUNO

$\mathrm{N}$-Soliton solutions to the DKP equation and Weyl group actions 